\title{
The effect of luminance variation on the apparent position of an edge
}

\author{
SERGIO RONCATO \\ Università di Padova, Padua, Italy
}

\begin{abstract}
A gray outline against a white (or black) ground appears to deviate when one of the divided regions turns into black (white). The direction of shift is not predictable on the basis of luminance profile and polarity contrast of this part of contour, called gray edge (to indicate a stepwise gradient from black to gray and from gray to white). Rather, it appears to depend on the luminance profiles of the collinear regions: A gray edge shifts toward the dark side whenever collinear with a gray line traversing a white ground. The same gray edge takes the opposite direction whenever it extends against a black ground. This rule proved to be successful in predicting the illusory convergence of the sides of a square that formed the stimuli of the first experiment, but the magnitude of the phenomenon was affected by luminance ratios and polarity contrasts of the gray edges, in agreement with the findings of the experiments on gray or blurred edge misalignment. A second experiment tested some hypotheses predicting the combined effects of two or more distorting sources. These hypotheses, suggested by the physical theory of vector sum, were partially disproved. A new model is proposed that assumes different ways of integrating local distortions. The third experiment tested predictions of how distorting pulses in opposite directions combine. The illusory misplacement of edge studied in this experiment is proposed as the underlying phenomena of the café wall illusion, the hollow square illusion, and other illusory phenomena observed with blurred areas. A connection with the induction grid phenomena is hypothesized.
\end{abstract}

Observers when shown a pattern such as that in Figure la report seeing small black squares adjacent to "perpendicular waving lines." All observers see the intersecting lines forming squares with swollen or concave edges. Swelling is reported whenever corners are occupied by black squares, whereas convexity is reported in the other cases.

Line waviness is an illusory effect since the pattern is made up of perpendicular straight lines and pairs of black squares joined at the corners and diagonally lined up (Figure $1 \mathrm{a}$ ) or with the corners overlapping (Figures $1 \mathrm{~b}$ and 1c). It seems obvious to search in these regions for the source of the illusory waving because the perpendicular lines, when bounding the edges of the small black squares, appear to bend as if the black area has an attraction strength on them. The characteristics of this distortion and the geometrical elements involved (i.e., a gray line separating black squares on a white ground) reminds us of a wellknown optical geometrical illusion, the café wall. The pattern depicted in Figure 1 a could be a particular version of the basic illusion studied by Gregory and Heard (1979), which is a limiting case in which the black tiles have the largest lateral displacement. Nevertheless, some experimental findings that failed to register an apparent slant of

This research was supported by a grant from MPI. The author thanks Clara Casco for her advice, encouragement, and review of this paper, Riccardo Massaro and Giovanni Caputo for valuable comments and help with computer programming, and this journal's referees for their constructive comments. Correspondence should be addressed to $S$. Roncato, Università di Padova, Dipartimento di Psicologia Generale, Via Venezia, 8, 35131 Padova, Italy (e-mail: roncato@psico.unipd.it). the mortar line in this condition, or "chessboard phase" (Haig, 1989; McCourt, 1983; Moulden \& Renshaw, 1979), discourage any analogy between the two phenomena. ${ }^{1}$ Further observations indicate that Figure la may be an example of a more general phenomenon we can observe when an outline borders regions of different luminance. Figure 1d is the best representation of this phenomenon, clearly observable in the form of apparent convergence of the horizontal edges of the rectangle.

All the perceived deviations seen in the configurations above seem concomitant with two geometrical characteristics of the gray line, part of which lies against a white background and the remaining part of which divides a dark region from a white one. Such perceived displacement could be related to the more general problem of the misperception of edge position.

It is well known that the precision with which the location of an edge may be discerned is affected by edge blur (Mather \& Morgan, 1986; Morgan \& Aiba, 1985; Watt \& Morgan, 1983) and luminance ratio between contour line and figure or background. Gregory and Heard (1983) observed apparent displacement of light and dark edges due to changes in background luminance. They measured the static displacement of two edged striped rectangles vertically aligned, discovering that a gray rectangle that has a dark strip on the left side and a light one on the opposite side appears displaced toward the right when the background luminance decreases. For increasing background luminances, the direction of displacement reverses. Similar effects were observed by Naiman and Makous (1993a, 1993b) in an edge-alignment experiment in which the 


\section{\%:}

a

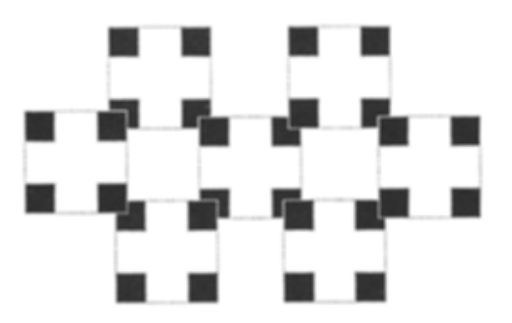

b
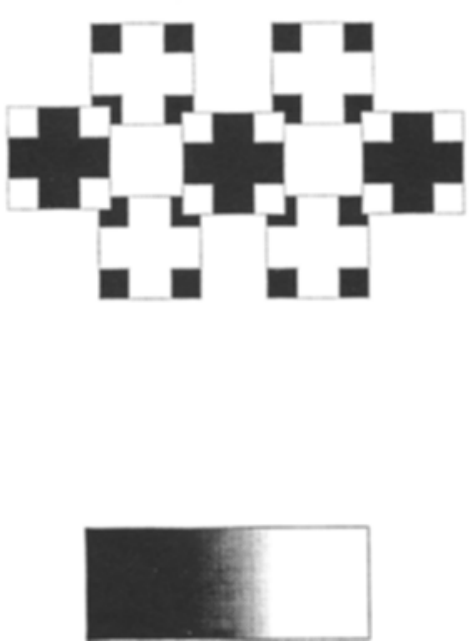

Figure 1. Some versions of the illusory phenomenon explored in the present research. All the squares, both larger and smaller adjacent to corners, are regular and bordered by straight lines.

observers had to adjust the position of a sharp edge in order to align it as closely as possible with the perceived gray edge - that is, a contour formed by a gray strip embedded between a black and a white region. The results showed a nonlinear relationship between the strip luminance and the perceived position that approached linearity as strip thickness decreased.

Some studies on the localization of blur edges have demonstrated that consistent misperceptions may occur. Mather and Morgan (1986) have found that reliable errors in the perceived positions are registered when ob- servers are asked to align edges of different blur widths or opposite contrast polarities. The error consists in shifting each edge toward its dark phase. This result, congruent with the prediction generated by the "irradiation hypothesis," has been confirmed by Bex and Edgar (1996), who observed that the misplacement of a blur edge toward the dark side is significantly affected by the edge contrast and the blur width.

Does the illusion illustrated in Figure 1d result from the same mechanisms underlying edge misperception? We intend to demonstrate that the line bendings in Figure $1 \mathrm{~d}$ are an unexplored illusory effect quite distinct from the gray or blur edge misperceptions, though it may be influenced by the same factors that give rise to this latter illusion. In order to support this belief with solid arguments, we must attain a clear description of the conditions in which the phenomenon occurs. This requires defining a number of terms clearly. Sharp edge means that the luminance gradient shifts abruptly from white to black. Gray edge (or fuzzy edge) means that the luminance gradient shows two steps, from black to an intermediate gray and from gray to white. Edge (space) blur means that a luminance gradient shows black shading into white according to a blurring function. Boundary contour (or outline contour) means that a line has luminance higher or lower than both the adjacent.

In Figure $2 a$, the basic stimulus pattern we used in two experiments is schematized. It was made up of a rectangular shape in which one can see a white central area fading to black in a symmetrical way, a gray contour and a white background. This pattern allows an observation of what happens when the same gray line is partly an outline (i.e., when it divides the two white regions) and partly an intermediate step in a luminance gradient (i.e., when it divides a black region from a white). The shading of black into white (blur direction) makes the contour deviations easier to detect but it has no effect on illusion direction and magnitude. By modifying its width, we may, for example, make different appearances of the illusory changes of directions evident: abrupt ones (in Figure 2b), called shifts hereafter, and progressive ones (in Figure 2c), hereafter referred to as tilt or slanting.

Along the contour of the horizontal side, one may distinguish two luminance profiles corresponding to the gray central outline and the lateral gray edges. Henceforth in this paper, the different luminance profiles will be indicated by the letters "B" (black), "G" (gray), and "W" (white), which, when read from left to right, represent the luminance arrangement of the inner area, the contour, and the ground. BGB profiles correspond to a gray outline against a black ground and WGB or BGW profiles correspond to gray edges. In Figures $2 b$ and $2 c$, the horizontal upper edge has a central WGW profile that changes to $\mathrm{BGW}$ in the extreme left and right segments. The same pattern against a black ground shows a central WGB profile flanked by two BGB profiles (Figure 2d). When the ground is of a gray lighter or darker than the contour, the 


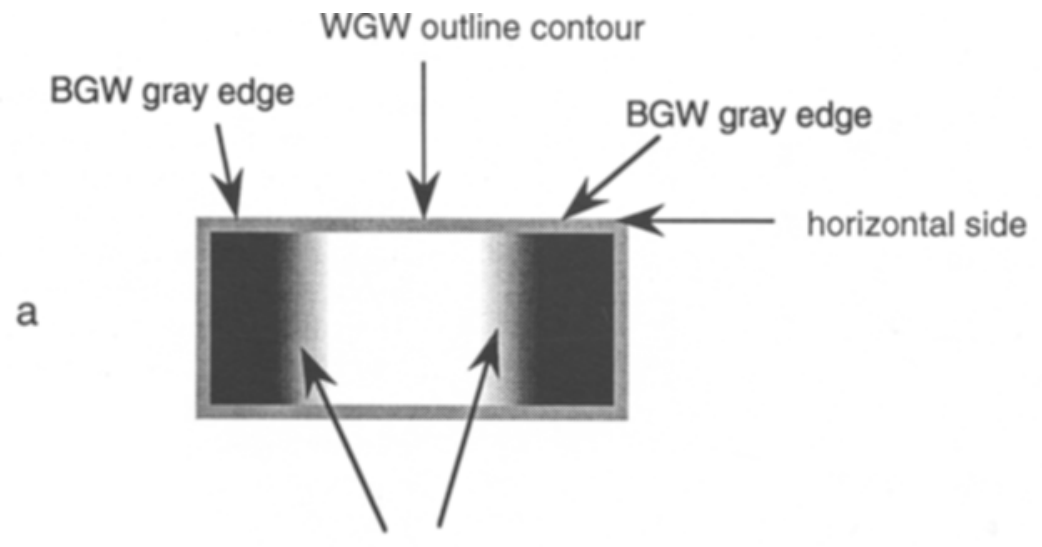

blur directions

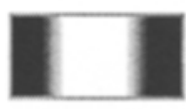

b

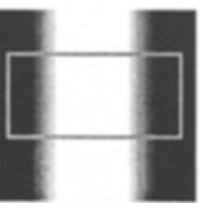

f
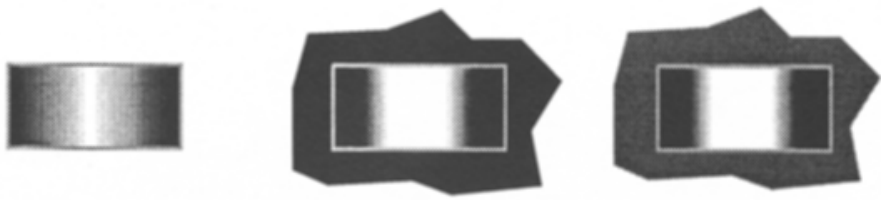

e

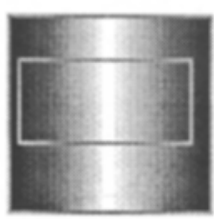

g

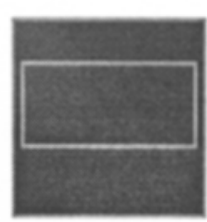

h

Figure 2. (a) Schematic representation of different luminance areas in the configuration stimuli used. This drawing illustrates the meaning of some terms often recurring in this paper. Note that gray (or fuzzy) edge stands for two abrupt changes of luminance: from black to gray and from gray to white. The uppercase letters are the initials of white (W), gray (G), and black (B), and they indicate the arrangement of the inner area, contour, and ground luminance. Shading has been obtained by linear increment in luminance. The contour of the inner square is mid-gray ( $50 \%$ black in the drawing). The illustrations in $b$ and $c$ show the effects of different width in space blur; $d$ and e show the effects of luminance decrease with respect to $b$. The illustrations in $f, g$, and $h$ are control patterns that were drawn so that the points lying on the same vertical line are isoluminant.

profile arrangements are the same as those observed with white and black grounds, respectively, even if the length ratio between the outline and the gray parts of the edge may change with larger space blur (Figure 2e).

Close inspection of the patterns in Figure 2 gives the first important suggestion for the inquiry into the origins of the Figure 1 illusory phenomenon. Two patterns, in particular (Figures $2 b$ and $2 d$ ), reveal very interesting il- lusory distortion of their contours. The two rectangular shapes appear to undergo two distorting effects in opposite directions: the gray edge shifts toward the dark side (Figure 2b), which leads to the apparent convexity of the rectangle, whereas the central gray edge shifts toward the white side (Figure 2d), giving rise to the apparent concavity of the rectangle. Note that the profiles of luminance of these two edges are identical, though different in po- 
larity contrast; therefore, there is no reason to expect that the same gray edge undergoes displacement into black in Figure $2 b$ and in the opposite direction in Figure 2d. Consequently, we are led to presume that the illusory effects originate in a region different from (or wider than) the space in which a gray line divides a black area and a white area. A more systematic observation may enable us to discover the factors responsible for the direction of the illusory effect. For this purpose, we constructed some patterns that allow an analysis of the effects of a larger number of combinations of profiles of luminance. The patterns (Figure 3) were derived from the basic one in Figure 2a by combining two symmetrical blur directions (central white fading into black, and vice versa), with black or white neighboring areas and two blur locations inside or outside the rectangle. If we explore the horizontal sides of the rectangle, we may easily distinguish the regions where the gray contour appears as an outline and where it borders a gray edge.

The observers quickly note that none of the rectangles appear as they are in reality (i.e., having a perfectly linear border). Rather, the horizontal sides are perceived as either concave or convex. These eight configurations allow a check of whether the illusory bending is dependent on systematic errors in perceiving the gray edge location. For this purpose, we must take into account that the same mid-gray outline was used to draw the contour of the eight rectangles and that black or white fill the adjacent areas.
Given these conditions, the same luminance profile generates the gray edges in the eight patterns of Figure 3. If this profile gives rise to a misperception in the edge location then the shift should always occur in the same direction (i.e., toward the dark side or toward the light side). An interesting observation can be made by comparing the patterns in which the gray edges have the same location and polarity contrast. The rectangles in $\mathrm{a} 3$ and $\mathrm{bl}$ are both centrally bordered by a gray (BGW) edge. Despite this, the central region appears to shrink in the former and widen in the latter (i.e., shift into the black in al and into the white in $\mathrm{b} 1$ ). In $\mathrm{a} 2$ and $\mathrm{b} 4, \mathrm{a}$ WGB gray edge borders the central part of the horizontal sides, and, again, distorting effects in the opposite direction are observed. The pairs of patterns $a 4-b 2$ and $a l-b 3$ are made up of rectangles in which the gray edges border the extreme horizontal sides. The comparison demonstrates that gray edges, having the same location and contrast polarity, appear to be shifted into the dark side in a4 and into the bright side in b2, into the white side in a1 and in the opposite direction in $b 3$.

There are strong grounds for concluding that concavity and convexity are illusory effects quite distinct from the edge-misperception phenomena, since the apparent contour deformations are observed in conditions of absolute steadiness of luminance profiles and luminance ratios. At present, we do not know which factors may be invoked to account for the direction of the illusory shift. a1

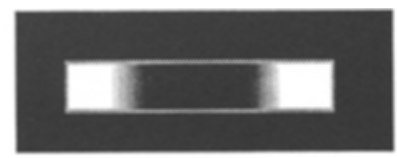

a3

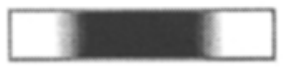

a2
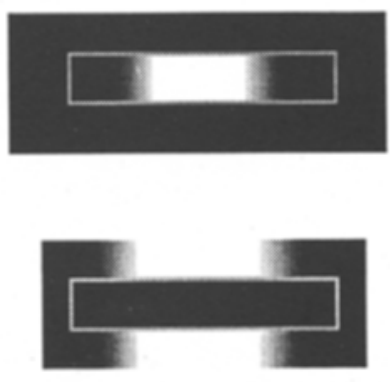

b2

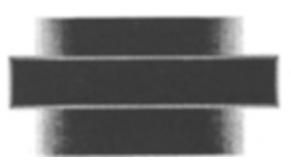

a4
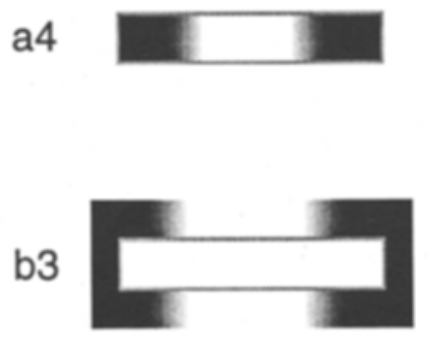

b4

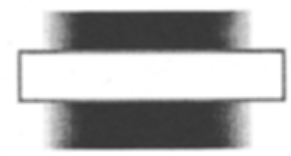

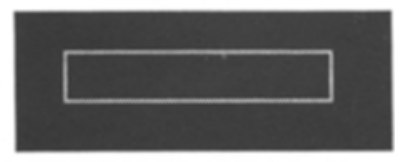
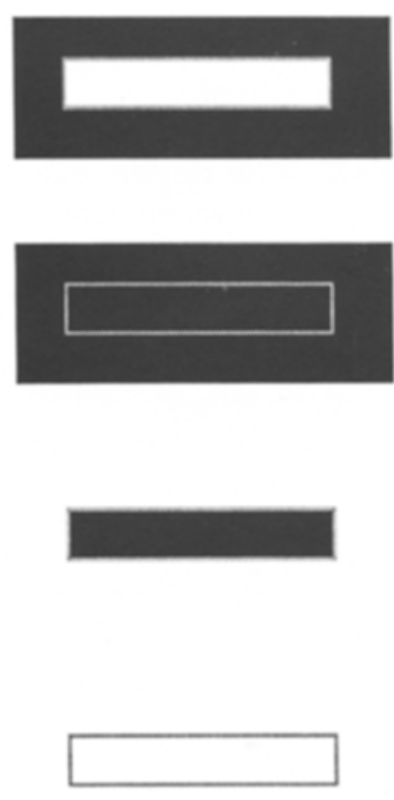

Figure 3. Patterns of variable luminance that illustrate the contour distortions of a regular rectangle against different types of ground. In the rightmost column, the rectangle is drawn with a homogeneous inner area against an isoluminant or nonhomogeneous background. The outline gray contour was mid-gray $(50 \%)$. The same blurred strips served to shade white into black in the rectangle area or the ground. Note the apparent concavity or convexity of the horizontal sides of the rectangle of the leftmost and central columns. 
However, the investigation has a fixed course because the sources of contour deviations must be searched for in the regions flanking the gray edge-that is, the regions where the outlines extend the gray edge. We may inspect the patterns in Figure 3 again and register which conditions are concomitant to apparent concavity versus convexity. A relevant regularity emerges: The shifts toward the dark side (white expansion) of the gray edge occur in patterns a3, a4, b3, and b4, in which only WGW outline profiles collinear with the gray edges are observed. The shifts toward the bright side are observed in al, a2, b1, and $\mathrm{b} 2$, in which the gray edges are flanked by BGB profiles. This relation between apparent shift direction and flanking conditions may be expressed in another form. If we follow an outline segment of both BGB and WGW luminance profiles and register toward which side it moves when one of the two adjacent regions changes in luminance, we may easily observe that it is this region that seems to attract it. For example, an outline gray segment dividing two white areas will appear to deviate if one of these becomes black--the direction it takes is toward the black.

This rule helps to clarify only one aspect of the illusory phenomenon (i.e., the direction it takes). Concerning amplitude, two types of factor must be taken into consideration. The first includes the "irradiation effect" and other factors that affect the perceived position of a gray edge. These factors are likely to act, enhancing or reducing the amplitude of the illusion, following the laws discovered in the above-mentioned research on gray edge misperception. A second type of factor becomes important when we consider different extensions of the region where the distorting factors arise. A gray edge that has an outline extension on one side may appear displaced differently with respect to an identical gray edge with outline extensions on both sides. Different configurations of this second type may be obtained by combining a gray edge with collinear and contiguous outlines. However, one configuration in particular raises an interesting problem: a gray edge that is collinear with a BGB outline at one extreme and with a WGW outline at the other. This is a condition in which the same gray edge is acted on by two distorting pulses: the former induced by the BGB outline that shifts the edge toward its white side, the latter induced by the WGW outline that displaces in the opposite direction. Will a distortion emerge in this condition and what direction will it take? Do the basic distorting effects sum so that an overall effect arises? Is this predictable in direction and magnitude?

In the present study, the last two experiments were devoted to systematically observing the combined effects of displacement affecting small or wider contour regions. In the first experiment, we concentrated on the observations in the simplest condition in which a gray edge and an outline interact - that is, a line that appears partly as an outline and partly as a gray segment embedded between a darker area and a lighter area.

\section{EXPERIMENT 1}

The aim of Experiment 1 was twofold. First, we intended to demonstrate that the direction toward which a gray edge appears displaced is predictable on the basis of luminance profiles of the aligned outlines (i.e., the polarity contrast between the gray line and background). Second, we aimed to check whether the amplitude of the illusory phenomenon is affected by factors acting only in the smaller region of the gray edge (i.e., the luminance ratio between the contour line and the divided areas).

In order to acquire illusion measures, simplified versions of Figure 3 stimuli were introduced. Examples of these stimuli, which reproduce half of the patterns in Figure 3, are depicted in Figure 4. They are made up of a square centered inside a circle and bordered by a gray outline. If either the circle or square shows change of luminance (black fading into white from left to right in Figure 4), then, in the horizontal sides of the square perimeter, a gray edge appears for some length and an outline appears for the remaining part. The conditions necessary for misalignment to arise are thus reproduced. In Figure $4 \mathrm{a}$, the left half of the horizontal side is a WGB gray edge, whereas the other half is bordered by a WGW outline. In Figure $4 \mathrm{~b}$, a BGW gray edge replaces the WGB of Figure 4a following the changes in shading location. By introducing patterns such as those in Figure 4, we may obtain all the combinations of luminance profiles analyzed in Figure 3 with the advantage of gaining the estimate of illusion amplitude in the form of perceived convergence or divergence of the horizontal sides. This estimate will be described below as an adjustment task.

\section{Method}

Subjects. Fifteen volunteers acted a subjects. nine were postgraduate students; the other 6 were experienced psychophysical observers.

Stimuli and Procedure. The stimuli were generated in a computer framestore (Matrox) with 8-bit resolution per gun and were displayed on a Multisync P750 NEC monitor at a frame of $85 \mathrm{~Hz}$. Screen resolution was $1,152 \times 864$ pixels. Gray values were linearized using a Minolta Luminance Meter LS100. The pixels on the screen were $0.25 \mathrm{~mm}$ in height and width, subtending nominally $0.42^{\prime}$ of arc at a viewing distance of $200 \mathrm{~cm}$. Luminance was in the range $2.2-106.5 \mathrm{~cd} / \mathrm{m}^{2}$. The randomization of stimuli and response acquisition were under computer control. Each display included the test stimulus and, $3 \mathrm{~cm}$ below, the comparison shape. The test stimuli consisted of a square $(200 \times 200$ pixels $)$ centered inside a circle 500 pixels in diameter. The outline contour of the inner square was 3 pixels thick, subtending a visual angle of $1.29^{\prime}$ of arc. The comparison stimulus consisted of a black-outline (1 pixel thick) isosceles trapezoid with the bases vertically oriented. The subject could adjust the convergence angle of the obliques until the trapezoid, or square, appeared to equal the test stimulus. At a visual distance of $200 \mathrm{~cm}$, the whole stimulus subtended a visual angle of $5.87^{\circ}$ of arc.

The experiment was in two blocks: one devoted to the matching task of a square against a shaded ground (shading outside), the other one devoted to the matching task of a shaded square area against a ground of homogeneous luminance (shading inside). The luminance gradient (blur direction) covered the entire width of the square. 


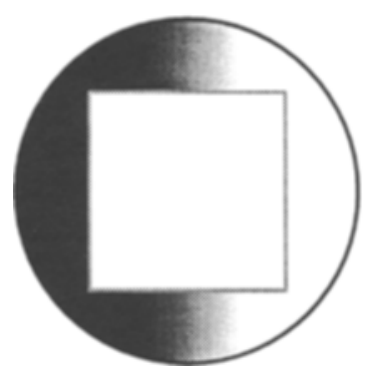

a

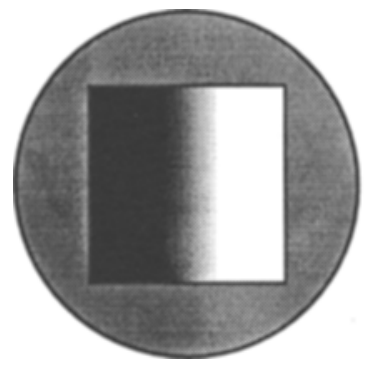

C

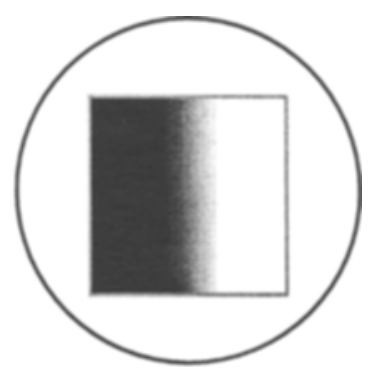

b

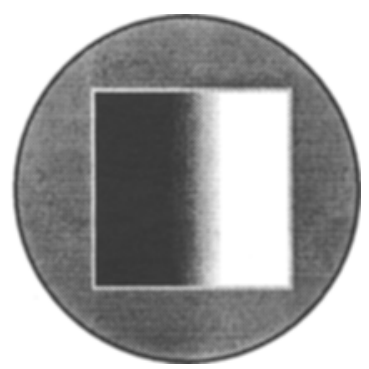

Figure 4. Some examples of the stimulus patterns used in Experiment 1. The shadings are representative of those used in the experimental stimuli, but they are exactly reproduced. Here, a thicker space blur has been drawn in order to make the outline part and the gray edge of the horizontal contour more evident.

Fifteen stimuli were constructed from the factorial combination of five levels of luminance of the square perimeter and three levels of luminance of the nonshaded area. The five perimeter luminances were $2.2,27.7,52.3,78.6$, and $106.5 \mathrm{~cd} / \mathrm{m}^{2}$. The luminance of the homogeneous area was set at one of three values: $2.2,52.3$, or $106.5 \mathrm{~cd} / \mathrm{m}^{2}$. Each of these 15 stimuli was presented in two configurations that differed in blur-direction location (inside vs. outside the square). Two sets of the resulting 30 patterns were prepared. The first had its white phase on the right, and the other had its white phase on the left. Ten control patterns were added. Some examples are presented in the rightmost column of Figure 5. They were constructed so that both the inner area and the outer area had homogeneous (though not necessarily equal) luminance or employed an outline square contour against a shaded ground.

The experiment was carried out in a dark room, and the subjects sat in front of a screen. Judgment of illusory convergence was obtained using the adjustment method. The stimuli were run in random order, and four judgments for each stimulus configuration were made.

To familiarize the subjects with the task and apparatus, 10 practice stimuli were used. A configuration was randomly selected from the set and was displayed; the experimenter then indicated the two horizontal sides of the test square and told the subjects that the task was to decide whether or not they were parallel. The subjects were then instructed how to adjust the trapezoidal shape to match the perceived convergence or parallelism of the test shape.
Each stimulus appeared showing the adjustable trapezoid with one base 15 pixels longer or shorter than the opposite base, according to a random computer sequence. The length of the adjustable bases could be increased or decreased symmetrically by 1 pixel. The " $>$ " key was used to lengthen the right base while, at the same time, shortening the left base. The "<" key had the opposite effect. Convergence of the obliques decreased or increased about $1.14^{\circ}$ (corresponding to $0.57^{\circ}$ of single oblique increment) with every keypress. The "return" key advanced the display. The subject continued to press the keys for as long as he/she wished, with free inspection of the stimuli. When the convergence of the two lines appeared equal, the subject pressed the "return" key, and the tilt (from the horizontal axis) of the upper side of the adjustable trapezoid was recorded.

\section{Results}

The matching data are reported in Figure 5 as the mean number of pixels required to symmetrically increase/ decrease the bases of the trapezoids. These data indicate the direction and magnitude of tilt, with regard to the horizontal, to which the oblique lines must be adjusted to reproduce the apparent convergence of the sides in the test figure. Positive values stand for clockwise tilt; 1 pixel corresponds to $0.57^{\circ}$ of tilt (for the first 10 units) 
INNER CONTOUR LUMINANCE $\left(\mathrm{cd} / \mathrm{m}^{2}\right)$

$\begin{array}{lllll}2.2 & 27.7 & 52.3 & 78.6 & 106.5\end{array}$

1
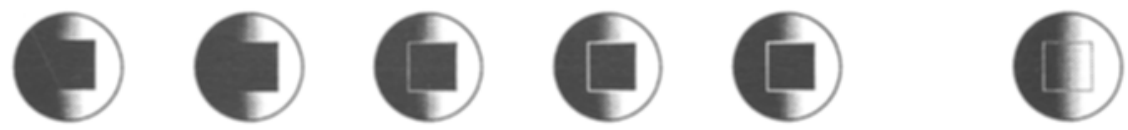

$.6(.25)$

$-7.2(1.14)$

$-6.6(1.21)$

$-4.3(1.36)$

$-1.9(1.31)$

2
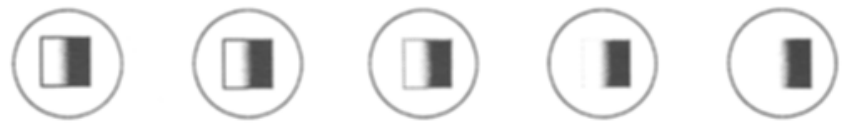

7.7 (1.04)

$8.2(1.38)$

$9.3(1.2)$

$9.8(1.52)$

$.4 \quad(.67)$

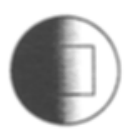

3
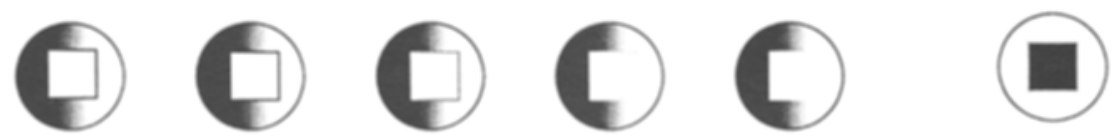

$7.6(1.23)$

$9.2(1.24)$

$10.3(.98)$

$10.7(.96)$

$2.8(.64)$
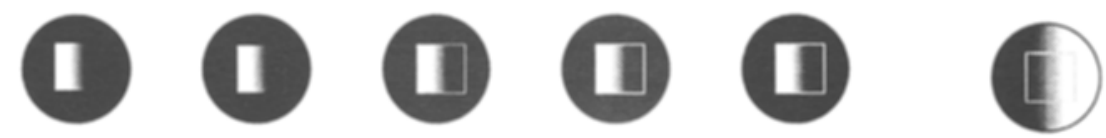

$2.5(.68)$

$-5.6(1.28)$

$-5.9(1.32)$

$-4.1(1.03)$

$-3.2(1.62)$
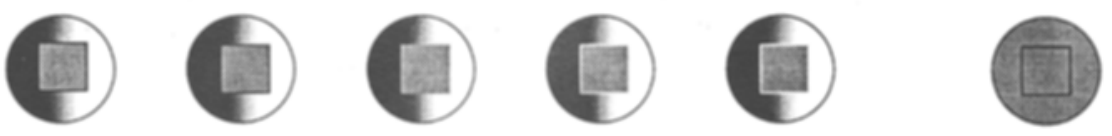

8.3(1.58)

9.9(1.43)

$.9(.60)$

$-7.2(1.81)$

$-4.5(1.39)$
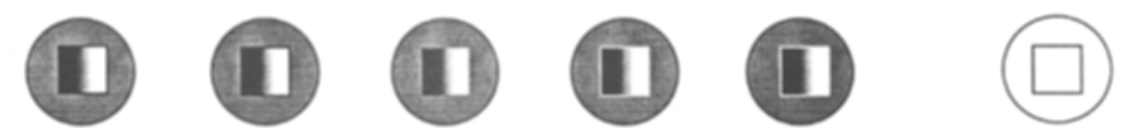

$-7.3(1.23)$

$-7.5(1.07)$

$-.9(.85)$

$7.4(1.61)$

$4.9(1.69)$

$.77(.12)$

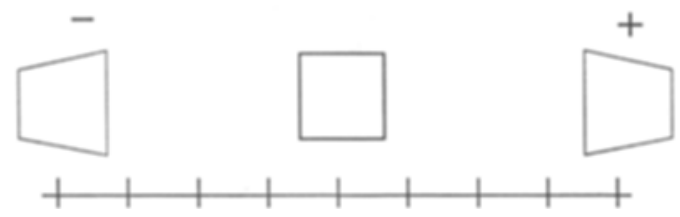

Figure 5. The mean matching data of Experiment 1, representing the mean symmetrical elongation in pixels of the left base of the adjustable trapezoid. Positive values indicate a clockwise tilt in the upper side of the trapezoid (see the bottom illustration). The values significantly different from the mean estimates to control patterns are in bold type. The standard error of the mean is indicated in parentheses. The stimuli are not exactly reproduced here, since a narrower blur direction has been inserted in order to render the outline and gray edges more evident. 
from the horizontal axis. Figure 5 shows the matching data for each combination of border and ground luminance. The judgments to opposite directions of shading were pooled together, since these did not differ statistically. The stimuli have been ordered in such a way as to allow the comparison of illusory effects produced by configurations in which the gray edges had the same location and polarity contrast. For example, in the first two rows of Figure 5, some patterns are reported in which the left half of the horizontal contour is (when visible) an outline and the right half is a BGW gray edge. Each vertical pair allows us to compare the illusion magnitude and direction in patterns having gray edges of identical luminance profile, location, and polarity contrast. The data of the first row were obtained by recoding half the judgments so that they appeared as if they were made to black shading rightward into white. Pooling in the opposite direction was carried out for the results of the second row.

The results for the 30 conditions were submitted to statistical analysis to check whether the mean convergence magnitudes were significantly different from the baseline control condition (i.e., the mean estimates given to the control patterns). The $t$ test gave significant results ( $p<$ .05 ) for the values indicated in bold type in Figure 5 .

Direction of gray edge shift (tilt). For the purposes of analysis, the judgments were divided into two parts: (1) estimation magnitude of patterns having black or white homogeneous areas, and (2) estimation magnitude of patterns having gray homogeneous areas.

Examination of the data of the central pairs of patterns (the rightmost and leftmost pairs contain a contourless square and, therefore, cannot be compared) reveals reliable differences. In the central pairs of rows 1 and 2, we can observe counterclockwise tilt for the upper stimulus and clockwise tilt for the lower. In other words, the illusory convergence observed in the stimuli of the first row is generated by a gray edge tilting toward the white side, whereas the illusory tilt observed in the second row moves toward the black side. The tilts in opposite directions can be found in the central pairs of rows 3 and 4 (remember that all the 10 configurations of these two rows are made up of a square bordered on its horizontal half by a WGB gray edge). The stimuli of row 3 show an apparent tilt of the square gray edge toward the black regions, whereas the stimuli of row 4 appear distorted in the opposite direction.

A second important finding is given by the matching data within each row, which vary in magnitude but not in direction (row 4's first pattern is the only exception). This implies that the contrast between luminance of the homogeneous areas and contour do not affect illusion direction. The idea that the factors determining the direction of the illusion must be sought in the wider region including the outline and collinear gray edge then gains support.

The results agree with the hypothesis that the direction of apparent shift of the gray edge is determined by the luminance profile of the collinear outline. In fact, the gray edge, when collinear with a BGB outline (rows 1 and 4), appears misplaced toward the white region; otherwise (collinear with WGW outline, as in rows 2 and 3 ), it shifts toward the black region.

Gray homogeneous areas. Analysis of the results reported in the last two rows is more complex because the perceived location of the gray edge varies according to the luminance ratio of the contour and the homogeneous area and thus is not constant within the stimuli of the same row. However, also in these cases, it is possible to demonstrate that the direction of tilt is predictable on the basis of the flanking outline profiles. For this proposal, it may be more useful to apply the rule "the gray contour deviates toward the region of luminance change." Let us check which deviations occur in the different luminance profiles present in the horizontal sides of the squares of rows 5 and 6 . In the central vertical pair, no apparent distortion occurs. In contrast, in the two pairs of patterns on the left side, the horizontal edges shift toward the black region. In contrast, in the two pairs of patterns on the right side, the shift moves toward the white region. That is, the gray homogeneous area bores into a darker region in some cases and into a lighter one in others. Nevertheless, a clear relation emerges when we observe the contour distortions into a wider context: The gray area always bores into the area changing in luminance. The generality of this relation may be easily checked if we follow the outline region of the horizontal contour and register the direction it takes when one of the neighboring areas changes the luminance. The outline segment (located in the right half of the horizontal edge in the first two columns of rows 5 and 6 and in the left half in the last two columns) always deviates toward the shading region.

The basic illusory effect. This set of data gives further support to the hypothesis that the illusion direction is predicted by the flanking conditions of the gray edge. The relation between illusion direction and the luminance parameters of both areas and contour can be illustrated by an image that renders such a relation easier to understand. The contour and neighboring regions may be thought of as if they were a water stream and the banks it flows between. The conditions in which the contour luminance is lower than the luminance of the darker region or higher than the lighter region are analogous to a situation in which the water level is below the banks. When the contour luminance is intermediate between the neighboring luminances, it is as though the banks have collapsed and the water has burst through the breach. The contour begins to shift when isoluminant with one of the bordering areas, thus bearing a resemblance to a river that overflows owing to its level rising over the bank. This metaphor will be referred to as the overflow rule from now on.

In Figure 6, two patterns have been depicted that allow the opposite direction of shift of the same gray edge (bordering the left half of the rectangle) and the flanking outline condition to be matched.

Gray edge and the magnitude of tilt. A rough observation of the results in Figure 5 indicates that the illusion 

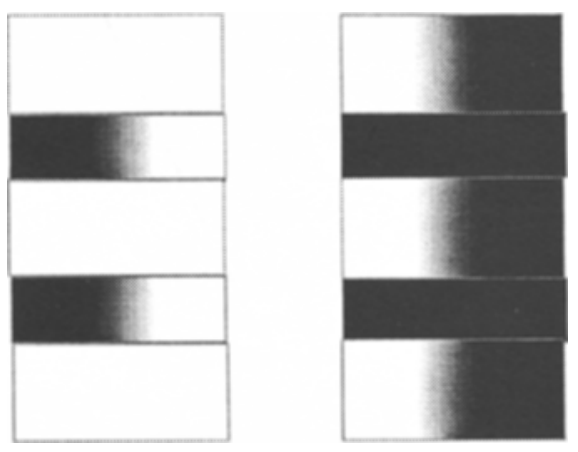

a

\section{b}

Figure 6. The same BGW gray edge and its detour when collinear with (a) a WGW outline and (b) a BGB outline. Note the opposite directions of shift in the two cases.

magnitude is higher when the homogeneous areas are white and when the square contour is very near in luminance to the homogeneous area. A deeper analysis of the results was carried out to ascertain whether the illusion strength is dependent on these two factors: the luminance of the homogeneous area and the ratio between this value and the luminance of the contour. This analysis requires a transformation of the experimental data in Figure 5 into mean "amount of distortion" (i.e., a measure of perceived tilt irrespective of its direction). These values were obtained by subtracting the mean perceived slant in the 10 control stimuli from the mean perceived slant in each experimental stimulus and by considering the absolute value of the difference. These values were analyzed in two distinct analyses of variance (ANOVAs). The first had as sources of variation locus of space blur (shading inside vs. shading outside), luminance of the homogeneous area (black vs. white), and luminance of the inner square contour (five luminance levels).

The strongest illusory effects were produced when the areas of homogeneous luminance were white ( 7.8 mean pixels vs. 4.8 pixels with black areas) $[F(1,14)=45.544$, $p<.001]$. This demonstrates that the tendency to shift toward the dark side was stronger. The effect of contour luminance was also significant $[F(4,56)=36.185, p<$ $.001]$. From black to white, the mean amounts of distortion were 4.8, 7.5, 8.15, 7.44, and 3.37 pixels. Post hoc analysis (Newman-Keuls) demonstrated that the illusory effect was weaker when the border luminances were $0 \%$ or $100 \%(p<.01)$.

This ANOVA yielded statistical significance for the interaction of contour and area luminance. This result will be analyzed later.

It is clear then that white irradiation affects the amplitude of the apparent convergence of the horizontal sides of the square. In some conditions (i.e., when the stimuli of rows 2 and 3 were presented), it enhanced the illusion; in other conditions (i.e., when the stimuli of rows 1 and 4 were presented), it weakened the illusory black area expansion.

A second ANOVA, carried out on the pattern of results of the last two rows of Figure 5, gave significant results $[F(4,56)=19.91, p<.001]$ for the factor levels of luminance. Analysis of the results and pairwise post hoc comparison (Newman-Keuls, $p<.05$ ) confirmed that the tilt of the gray edge was consistent both in the direction of the dark side and in the opposite direction; the former, however, yielded stronger results when the contours had high luminance.

White irradiation showed its effect not only in the configurations in which the gray edge was clearly visible but also in conditions in which the contour disappeared. The squares in the rightmost stimulus of row 3 and the leftmost stimulus of row 4 were perceived as converging, contrary to our expectations but in agreement with the "irradiation effect," because the clockwise tilt of horizontal edges may have been produced by the white boring into black. Note that, in the other contourless squares (leftmost and rightmost stimuli of rows 1 and 2), no expansion of black into white was observable. Pairwise post hoc comparison (Newman-Keuls, $p<.05$ ) confirmed that the contourless squares of rows 3 and 4 appeared more distorted than the corresponding squares in rows 1 and 2.

Illusion magnitude and luminance contrast. Stronger effects were observable with low contrast between the outline contour and the homogeneous area. To check how relevant this factor was, we carried out further analyses on collapsed data. Since our interest was to discover the relation between apparent slant and contrast between contour and homogeneous areas, this luminance ratio $(D)$ was calculated: abs $(L$ contour $-L \min ) /(L \max$ $-L \mathrm{~min}) . D$ was calculated for each of the 30 conditions, resulting in five groups: $0, .25, .50, .75$, and 1 . The results of the condition within each group were averaged, and the resulting five mean illusion magnitudes $(2.1,8.5$, $7.5,6.5$, and 6.1) were submitted to one-way ANOVA, which gave significant results $[F(4,56)=23.33, p<.001]$. Post hoc pairwise comparisons (Newman-Keuls, $p<$ .05 ) revealed that $D$ yields the strongest effects when its value is .25 ( 0 value yields the lowest minimal effects), a value that is significantly higher than .75 and 1 . This result is in agreement with the findings on gray edge perception, which demonstrates that its misplacement is a function of strip luminance. However, our data are not suitable for further analysis to test whether or not the trend approaches linearity.

\section{Discussion}

Three factors may be implicated in the apparent edge misalignment we have analyzed here: the irradiation effect, lateral displacement of areas toward low-contrast borders, and some unknown effects that have been de- 
scribed by means of the "overflow rule." The first two originate with the gray edge; the latter originates in a region that includes the gray edge and its extension as an outline. ${ }^{2}$

The hypothesis that the direction of illusory misplacement is dependent on its flanking conditions has strict implications. When, as in this experiment, only one side of the gray edge is flanked by an outline, the deviation may be predicted by the overflow rule; however, when the gray edge is collinear with two outlines at both sides, there may be contrasting "inductions." In particular, one has to understand what happens when the two flanking conditions have opposite luminance profiles (e.g., BGB and WGW) and consequently exert distorting powers in opposite directions on the gray edge. In order to make some testable predictions, we have assumed that this "power" or "distorting induction" can be assimilated to a force, something similar to an impulse that is given to a physical body. In this way, the distorting power can be represented by vectors, and the predictions of the overall effects will take the form of models of force composition (or sum). In Figure 7, an attempt has been made to predict the overall distortions arising from different combinations of central gray edges and flanking outlines, and vice versa.

This description derives from the previous findings and new assumptions. We assume that the distortion sources are to be sought in the gray edge rather than in the sharp region of the contour, because, whatever the distorting effects that may arise in the neighboring areas, they balance when the contour divides areas of equal luminance. Second, we assumed that, in the case of abrupt changes of luminance, a constant distorting force acts on the entire length of the gray edge and in a direction perpendicular to it. Consequently, the illusory effect can be represented as a vector, having the midpoint of the edge

a)
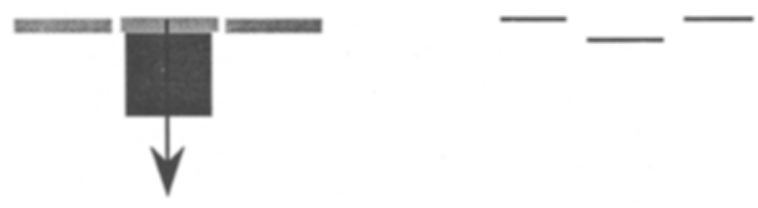

b)

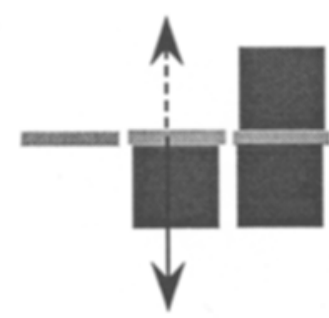

c)
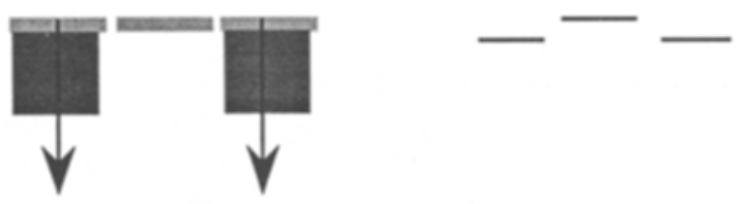

d)

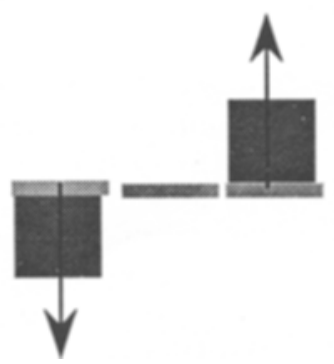

Figure 7. Different outcomes of distorting pulses. In the leftmost column, different combinations of gray edges and collinear outlines are presented along with the distorting pulses (indicated by arrows). Continuous-line arrows represent tendency to shift toward the dark side of the gray edge; dashed arrows represent shift in the opposite direction. The resulting shifts are represented in the second column. See the text for the assumptions that lead to this type of representation. 
as the application point and the perpendicular as application line. The vector direction is determined by the luminance profile of the flanking outline (i.e., by the overflow rule). Since the previous findings demonstrated that the shift toward the black side is stronger than the shift toward the white, the vector magnitude is expected to increase when the flanking outline is of a WGW type.

In Figure 7, different combinations of gray edge and collinear outlines have been explored in order to predict the overall effect of distorting forces summation. When, as in Figure 7a, the gray edge is flanked by outlines having the same profile of luminance, distorting forces are induced in the same direction. The arrow symbolizes the total amount of distorting power on the gray edge. This does not occur in Figure $7 \mathrm{~b}$, whose gray edge is acted on by concurrent forces induced by the WGW and BGB flanking outlines. They are represented by two vectors of different magnitudes owing to the stronger force toward the dark side. The difference in length is proportional to the difference in mean magnitude illusion produced by the two apparent tilts, toward the dark side and toward the light side, as inferred from the findings of Experiment 1. In Figures $7 \mathrm{a}$ and $7 \mathrm{~b}$, the central gray edge is expected to appear misaligned toward the dark side, but with weaker effects in Figure $7 \mathrm{~b}$ owing to the contrasting force in the opposite direction. The vector summation in Figures $7 \mathrm{c}$ and $7 \mathrm{~d}$ leads to predicting deformations in the form of contour concavity or rotation. We have no reason to expect differences in magnitude of the perceived misalignment in Figures $7 \mathrm{a}$ and $7 \mathrm{c}$, but two occurrences must be taken into consideration. If the flanking conditions affect both the direction and the strength of a shift, then the central gray edge in Figure $7 \mathrm{a}$ underlies a double pulse, and a stronger misalignment should be perceived. If the vector strength is not affected by the number of flanking outlines, then Figures $7 \mathrm{a}$ and $7 \mathrm{~b}$ have the same outcomes. Figure 7 illustrates this latter occurrence.

In conclusion, the present experiment has made the basic illusory effect evident (i.e., the apparent displacement of a gray edge when it is collinear and contiguous to an outline). In these conditions, the simplest ones giving rise to the illusory effect, the direction of misplacement is inferred from the overflow rule. However, in order to account for the apparent distortions that may originate in more complex conditions, one has to predict how the basic effects sum or interact. To attain this goal, a model of forces summation is proposed, and its predictions were tested in Experiment 2.

\section{EXPERIMENT 2}

In Experiment 2, we intended to test a complete set of predictions that may be derived from the model of forces composition illustrated in Figure 7. To attain this goal, it is necessary to check the overflow rule against situations more complex than those studied in the Experiment 1. In particular, it should be possible to observe what happens when the same area is bordered by different combinations of gray edges and outline contours. The uppermost pattern drawn in Figure 8 is very useful to this end. It is composed of four types of area that can be black or white: the central square (a), corner squares inside the central square (b), rectangles outside the central square (c), and ground (d). If the contour of the inner squares is gray, it may result in different combinations of fuzzy and outline segments. In Figure 8, where some examples of these patterns are illustrated, it can be seen that the sides of the inner squares may be defined by an outline contour (Figure 8, top) or may be entirely fuzzy (1) or made up of alternate outline and fuzzy segments $(2,3,4$, and 5 ). On the basis of the hypotheses of basic effects summation illustrated in Figure 7, we are able to predict some types of distortion that will arise along the contour; their directions are indicated by arrows in the configurations in the leftmost column of Figure 8.

The deformed effects resulting from vector summation are shown in the central column of Figure 8. Two equal vectors acting on the two extreme segments of the edge should give rise to apparent convexity if directed inward (row 2) and to an apparent concavity if directed upward. The apparent concavity of the square edge is the predicted result of a vector acting on the central segment of an edge with an inward direction (row 3). The same vectors acting in the opposite direction should produce an illusory convexity. The vector analysis illustrated in Figure 8 allows some predictions regarding not only the type of apparent deformation but also to its magnitude. In fact, the distorting vectors describe basic effects that may combine to produce stronger illusions. For example, a central inward vector combined with two lateral outward vectors may give rise to a stronger concavity illusion (row 4). More subtle predictions can be drawn if we create a simple typology of patterns. The 16 patterns that result from the different combinations of the four surfaces (a, b, c, and d; either black or white) can be distinguished in four groups, each leading to different predictions of illusion direction and strength.

Group 1: Patterns in which the edges of inner squares are outline contours. An example of these configurations is the uppermost pattern of Figure 8. No distortion is expected.

Group 2: Patterns in which the edges are entirely fuzzy. An example is the pattern in row 1 in Figure 8. No distortion is expected.

Group 3: Patterns in which the central part is occupied by an outline segment (or fuzzy) and the other parts are fuzzy edges (or outline contours). Examples are patterns 2 and 3 of Figure 8. Apparent distortion is expected as an effect of lateral vectors, such as those acting on the inner squares of patterns $3,5,10$, and 13 of Figure 9 , or a central vector, such as the one acting on the inner squares in patterns $7,8,14$, and 16 of Figure 9 . Our assumptions do not make it possible to predict whether the distortions concentrated on a central region are stronger than the same 


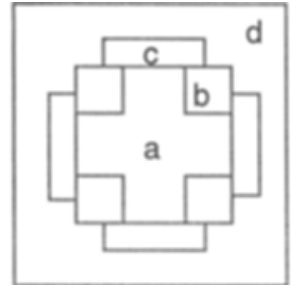

1

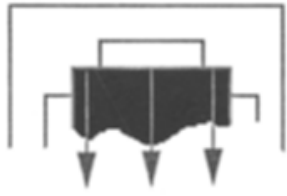

2

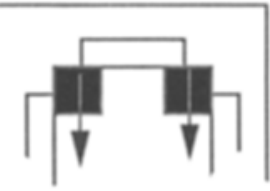

3

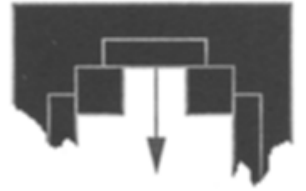

4

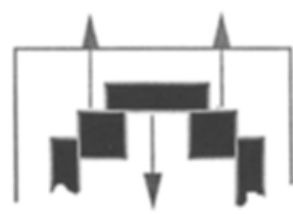

5

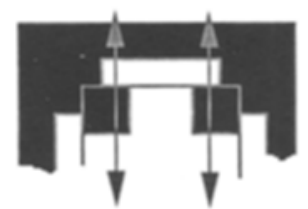

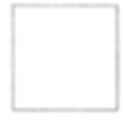
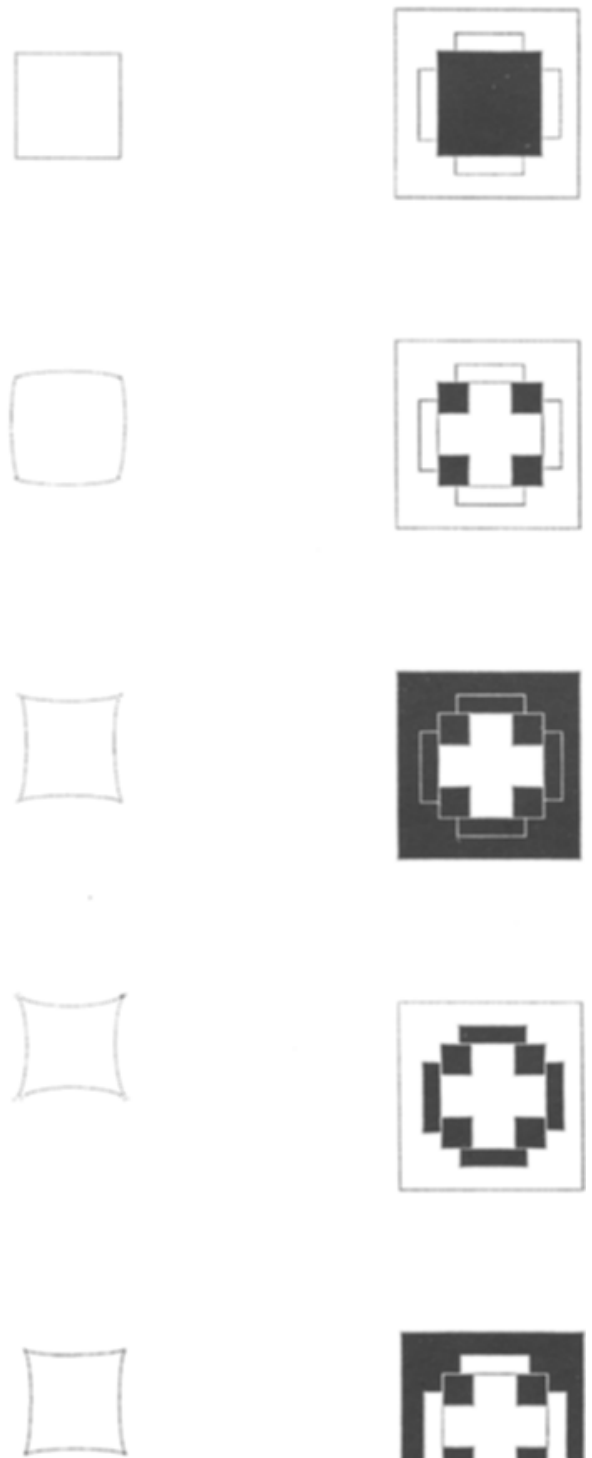

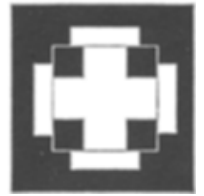

Figure 8. Distorting forces acting on the gray edges of a pattern (top shape) when its parts are filled with black and white at different combinations (leftmost column). The central column presents the expected apparent contour of the inner square. The rightmost column presents some examples. Row 5 represents the contrasting vectors acting on the two gray edges. The resultant vector is collinear with these edges, directing inside the square since this is the direction of the stronger vector toward the dark side. Note the apparent convexity of the example in row 5 , in contrast to the predicted concavity. 
distortions when distributed over two lateral regions. It will be the mean perceived distortion in these two sets of stimuli that provides an answer.

Group 4: Patterns in which the inner square contains more than two gray edges or outline segments. This type of configuration gives rise to pairs of opposing forces and to a rotational resultant. An example is the pattern in row 4 of Figure 8 . The magnitude of distortion resulting from the summation of these vectors is greater than the illusory effect one expects in Group 3, owing to the joint effect of two rotatory pairs. Compare, for example, the two patterns in rows 3 and 4 . The first is deformed by a central inward force; the second is deformed by the same force combined with two lateral opposite vectors. Consequently, the patterns in Group 4 are expected to be perceived as more distorted than those in Group 3.

The lowermost pattern of Figure 8 (row 5) belongs to the fourth group, but an analysis of the distorting effects leads to different predictions. As one can see, the two extreme outline segments (BGB) shift the gray edge toward the white side, whereas the central outline (WGW) gives rise to a force on the same gray edge having an opposite direction. This combination of gray edge and flanking outlines corresponds to Figure $7 \mathrm{~b}$, which illustrates the summation of two vectors having the same point and line application but opposite directions and different strength. Since it led to the expectation of a weak shift of the gray edge toward the dark side, the predicted illusory distortion produces a square concavity but of a small magnitude.

The 16 patterns generated from the basic ones in Figure 8 , by combining black and white surfaces, will be referred to as the experimental patterns. They were used as a basis to construct 5 other stimuli, indicated as the control stimuli. Each was derived from an experimental stimulus by replacing white areas with mid-gray areas and the contour with a light gray one or else by replacing the black areas with mid-gray and the mid-gray contour with a dark gray contour. In both cases, the perimeter of the square had a luminance lower or higher than the luminance of the adjacent areas-that is, a condition in which the contour is given by an outline. In these conditions, no illusory distortion is expected, since we assumed that the necessary condition for an apparent shift to arise is the contiguity of a gray edge and an outline segment.

\section{Method}

Subjects. Fifteen volunteers acted as subjects. Four were postgraduate students; the other 11 were experienced psychophysical observers.

Stimuli and Procedure. A total of 21 patterns were computergenerated (McDraw Plus) and displayed. The experimental pattern appeared on the center of a screen having an image resolution of $800 \times 600$ pixels ( 3 pixels $/ \mathrm{mm}$ ). It was made up of an outer square $(7 \mathrm{~cm})$, inside which was centered a smaller square $(4 \mathrm{~cm})$. Adjacent to its corners, four squares $(1.2 \mathrm{~cm})$ were drawn inside and four rectangles $(2.6 \times 0.7 \mathrm{~cm})$ were drawn outside so that the longer side was superimposed on the external perimeter of the inside square midway between the extremes.

A line of constant thickness ( 3 pixels for all stimuli) was used to draw the contour of all shapes. Its luminance was $43.8 \mathrm{~cd} / \mathrm{m}^{2}$, ap- proximately the intermediate value between the luminance of black areas $\left(1.93 \mathrm{~cd} / \mathrm{m}^{2}\right)$ and the luminance of white areas $\left(91.1 \mathrm{~cd} / \mathrm{m}^{2}\right)$. The luminance of the white and black regions were measured by a Minolta LS 110; a close-up lens was attached to the meter for taking measurements of the contour lines, specifically of the thickness constancy of (a) square contour in Figure 8. This last inspection was repeated for the whole set of 20 stimuli.

The judgments of illusion magnitude and direction were given on a 9-point scale, which appeared $4 \mathrm{~cm}$ below the pattern (see Figure 9 bottom). Point 1 corresponded to a square with convex edges; point 9 corresponded to a square with concave edges. The central point (5) was associated with a regular square, indicating that no contour distortion was seen, whereas points lower or higher than 5 were used to indicate perceived concavity and convexity, respectively, as illustrated by the shapes drawn below points 1 and 9 . The whole display and contour line subtended $3.72^{\circ}$ of arc and $1.70^{\prime}$ of arc, respectively, at a viewing distance of $200 \mathrm{~cm}$.

The subjects were tested individually in a dark room. They were familiarized with the range and variety of the stimuli and with the use of the judgment scale. Specifically they were told that their task was to examine the central region of the stimulus and to evaluate whether the contour indicated by the experimenter appeared as a square or a squared shape made up of concave or convex edges. After 5 practice stimuli, they were instructed to report their evaluations on a scale for each of the 21 stimuli randomly presented in two blocks.

\section{Results}

In Figure 9, the 16 experimental patterns and 2 control stimuli are presented, along with the mean estimations of illusion magnitude. In parentheses, the standard errors are reported. Below the two control stimuli, the mean estimate is reported. It was calculated by averaging the mean estimate of the distortion of the 5 control patterns. An analysis, using a $t$ test, was carried out to compare the mean illusion magnitude of the control condition. In Figure 9, the values significantly different from baseline control $(p<.05)$ are indicated in bold type.

In order to check these data against the predictions, it is necessary to bear in mind that no distortion is expected when the inner contour is entirely fuzzy (patterns 4 and 11) or outline (patterns 1 and 2 and the controls). Contrary to expectations, a weak concavity is perceived in patterns 4 and 11 . The gray edge shifts toward the dark side as predicted (because it is flanked by a WGW outline) in patterns $3,7,8$, and 13 , and it shifts in the opposite direction as expected (because it is flanked by a BGB outline) in patterns 5,14 , and 16 . The illusory effect is absent in pattern 10, contrary to expectations. Patterns 6 and 12 appear distorted as predicted (see Figure 7). Moreover, a very interesting disproof of the model of forces composition illustrated in Figure 7 is found in the results obtained with patterns 9 and 15 of Figure 8. In contrast to the predictions, both of these patterns appear strongly distorted. In addition, a convex shape is perceived in pattern 15 , whereas the model suggests that an apparent concavity is to be expected (see Figure 8, row 5).

The results were analyzed further to check the predictions on the magnitude of illusory effects as a function of the number of distorting vectors. Within the experimental stimuli, three sets were distinguished following the criteria discussed above: a set of patterns in which the inner 
1

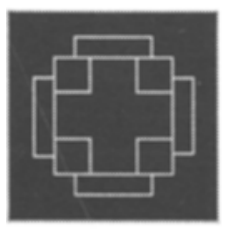

$5.0(.05)$

5

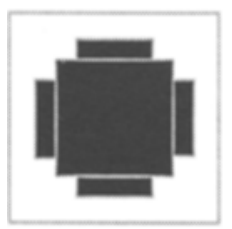

$7.1(0.22)$

9

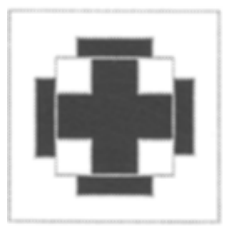

$2.2(0.16)$

13

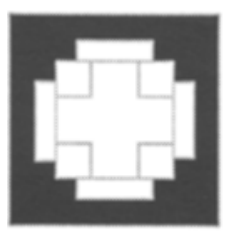

$6.9(0.23)$
2

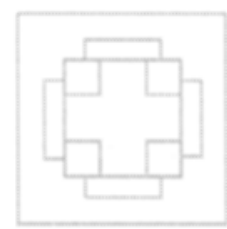

$5.0(0)$

6

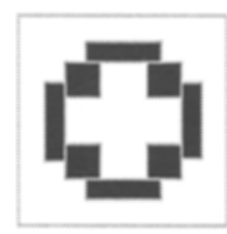

$7.9(0.26)$

10

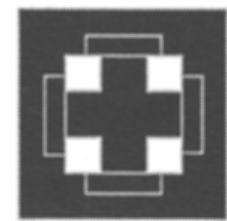

$5.03(0.25)$

14

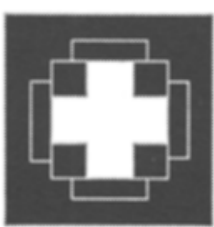

$5.4(0.12)$
3

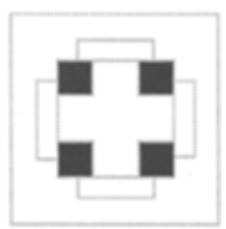

$4.1(0.31)$

7

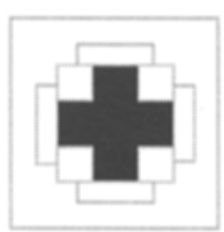

$6.0(0.17)$

11

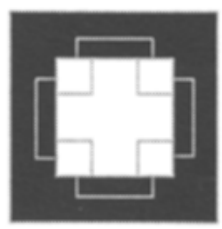

$5.5(0.12)$

15

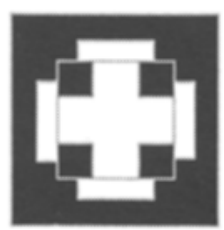

$2.8(0.23)$

controls
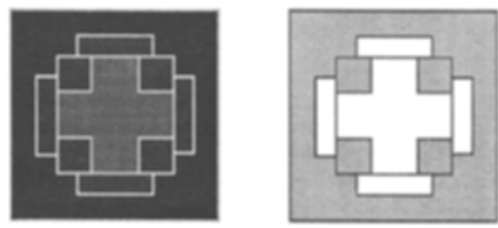

4

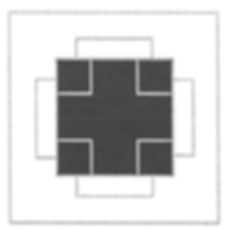

$5.6(0.16)$

8

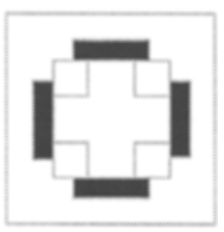

$3.1(0.28)$

12

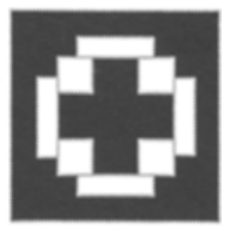

$7.8(0.22)$

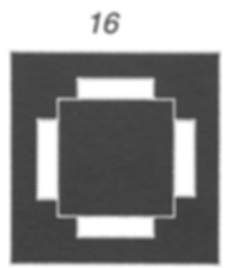

$4.3(0.12)$

$5.02(0.18)$

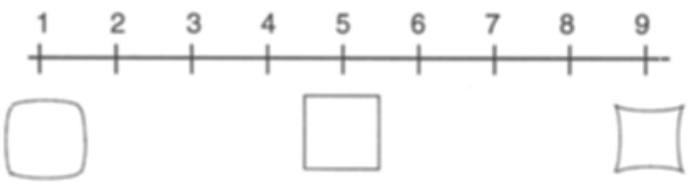

Judgment scale

Figure 9. Mean distortion estimates for the experimental and control patterns of Experiment 2 . The judgment scale is presented at the bottom of the figure. The values significantly different from the mean estimates to control patterns are in bold type. The standard error of the mean is indicated in parentheses. In this figure, the contour thickness and shade of the stimuli could not be reproduced exactly. 
square had entirely outlined or entirely fuzzy sides, a set having sides with a fuzzy or outline central segment, and a third set with each square side having two fuzzy or two outlined segments. As in Experiment 1, we converted each estimate in difference (absolute value) from the control condition (5.02).

The mean sizes of the illusion calculated in the three sets of stimuli are the following: 0.28 when the inner frame is entirely outlined or fuzzy, 1.24 when each edge has a central fuzzy segment flanked by gray ones or a fuzzy central segment flanked by two outlines, and 2.67 when the same edge had two outlined segments and two fuzzy ones. An ANOVA (and post hoc comparisons) demonstrated that the differences between the three means were significant $[F(2,28)=158.57, p<.001]$.

The present results confirm that the illusory shift toward the black side of the gray edge (1.45 mean magnitude illusion) is stronger than the shift in the opposite direction ( 0.77 mean magnitude illusion). The effect was statistically significant $[F(1,14)=10.04, p<.005]$. The location of the gray edge (central vs. bilateral) did not affect the illusion strength: The difference between the two means $(1.03$ and 1.22$)$ was not significant $[F(1,14)=$ $1.78, p>.05]$. This demonstrates that a gray edge flanked by two outlines does not underlie stronger misplacement with respect to the same gray edge flanked by an outline only.

\section{Discussion}

Experiment 2 was used to check two predictions. The first is generated by the overflow rule and states the direction of apparent slant when a fuzzy edge continues in an outline contour, or vice versa. The second is based on some assumptions of how the distorting vectors may sum. The predictions schematized in Figure 8 were largely confirmed. The exceptions concern patterns 4 and 11 in Figure 9 that unexpectedly appeared as concave, pattern 10 disconfirmed the predictions of concavity, and patterns 9 and 15 whose perceived distortion resulted opposite to the predicted one. Some subjects observed that the corners of both white and black squares in patterns 4 and 11 appeared as acute angles-a weak effect but sufficient to generate an overall impression of edge concavity. This effect reminds one of the "corner effect" analyzed by Moulden and Renshaw (1979). The lack of illusory effects in pattern 10 may be attributed to the white irradiation balancing the convexity effect; in fact, when the two factors act in the same direction, as in pattern 3, a consistent illusory convexity is perceived.

Two findings remain unexplained: the apparent convexity perceived in pattern 15 of Figure 9 and the magnitude of the illusory effects perceived in patterns 9 and 15 that are close to the maximum of illusion observed in this experiment. These results clearly suggest that the integration of local distortions cannot be accounted for by the simple model of Figure 7. To test an alternative model, Experiment 3 was carried out.

\section{EXPERIMENT 3}

The aim of Experiment 3 was to propose a model of integration processes to give a full account of the overall effects observed in Experiment 2 that do not obey the expectations based on what we know about forces summation. Let us again consider the local basic effect. It consists of an apparent detour of a contour line when the change of luminance of one of the neighboring regions renders it a line of intermediate luminance. This detour appears like a "step" when the change of luminance is abrupt. Otherwise (i.e., with a smooth gradient), it appears like an arc describing a deviation. When two or more of these local distortions occur on the same line, do they integrate? The answer can be found in some theories of the Mürstenberg illusion (Gregory \& Heard, 1979; McCourt, 1983; Morgan \& Moulden, 1986), according to which a series of local tilted elements gives rise to an overall slant effect. But they assume that the overall effect is produced when the number and length of local elements are consistent. Experiment 2 demonstrated that some sort of integration also arises in narrow areas where one or few edge misplacements arise. These integrations will be referred to as local integrations in order to distinguish them from overall integrations. In Experiment 3, a new model of local derivative effects was tested. This model is based on the assumption that the local integrations may proceed along different ways. Two of these operations are schematized in Figure 10. The first type of integration (Type I) can be observed in a configuration made up of a straight line collinear with the edge of two lateral squares: one above and the other below the line (Figure 10a). The central segment of the horizontal line is an outline segment flanked by two gray segments. Two opposite forces (represented in Figure 10a by two arrows) act on these gray edges. What is the outcome of the two distorting pulses represented by the two arrows? According to a vector model, the entire configuration underlies a counterclockwise rotation due to the "lever" arm of the pair of vectors. However, in addition to this suggestion, which allows the direction of apparent tilt to be predicted, other assumptions are needed. Given that the same vectors are constant, different distortions may appear. Two of these distortions (represented in Figure 10a by the dashed lines $i$ and ii) are of particular interest because they show different locations of the distorting effect (i.e., on the central outline region or on the two flanking gray edges). The former effect (i) could be expected when the central outline contour is shorter than the flanking gray edges, as the uppermost example shows. If this length ratio is reversed (i.e., the outline segment is longer than the flanking gray edges), then the apparent tilt of these extreme regions can be expected. This effect (ii) is illustrated in the rightmost column (the second example). In conclusion, the Type I integration consists in the apparent tilt of the central region of a segment or its extreme regions. The direction of tilt is determined by the rotatory pair of forces. 
TYPE I

(a)
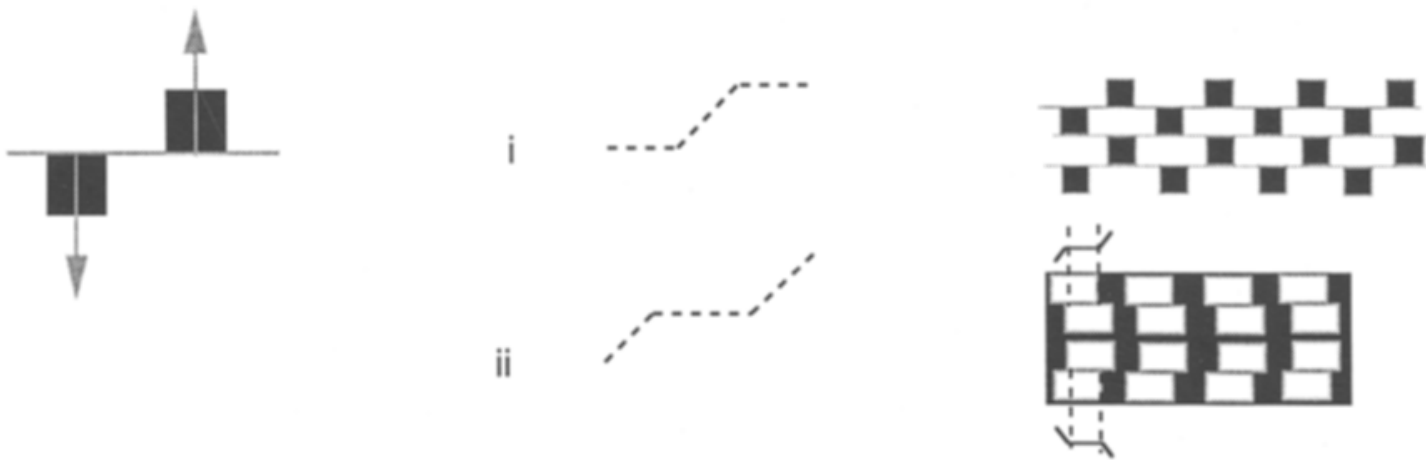

TYPE ॥

(b)
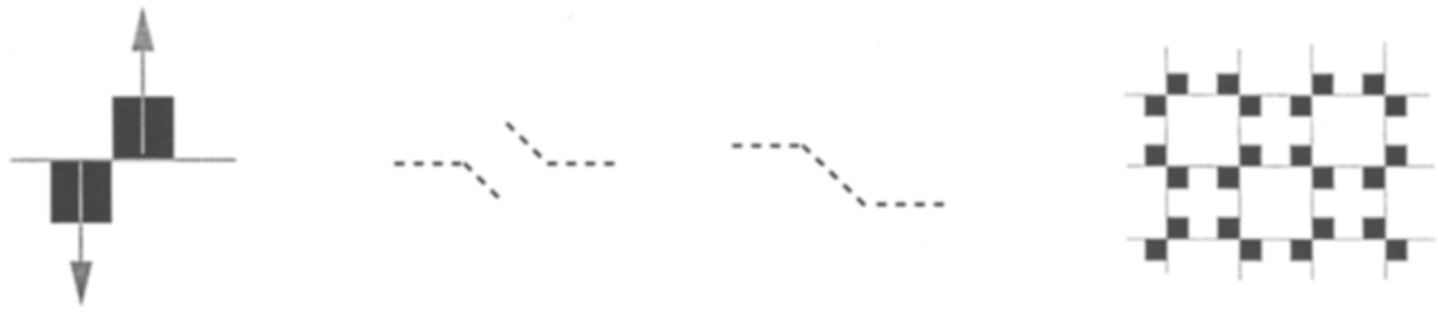

TYPE I + TYPE ॥

(c)

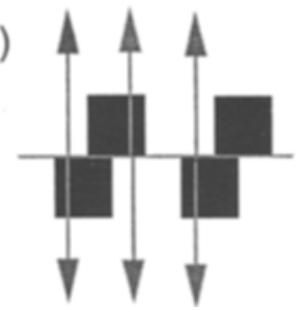<smiles>CCCCCCCC</smiles><smiles>CCC(C)C</smiles>
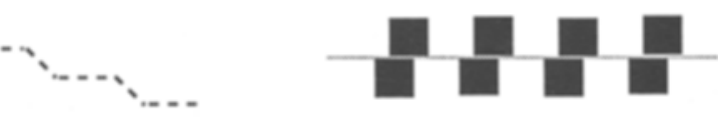

Figure 10. A schematization of how local distortions are integrated. Two patterns of distortion are (a and b) and their combined effects (c) are presented. In the central columns, the dashed lines highlight the effects attributable to the distorting forces and how they locally sum. The patterns in the right column serve as a preliminary check of the predicted illusory distortions. 
A pair of opposing vectors may even combine according to rules different from the one just considered. One of these particular cases (Type II) is given in Figure 10b. Here, the only difference with respect to Figure $10 \mathrm{a}$ is that the two squares are contiguous so that the two vertices meet. This leaves the vectors in Figure 10a and their directions unaltered, so there is no reason to expect illusory rotations different from those resulting in Figure 10a. On the contrary, the apparent tilt of the line passing between the two squares in Figure 10b takes a direction opposite to that in Figure 10a. This is easy to ascertain because it consists of the illusory effect described in the introduction (see Figure 1).

To explain why this occurs, two assumptions are needed. First, the two opposite vectors, acting on each gray edge, are displaced into the dark phase and are therefore pushed apart. This should produce a split in the dividing line, the breaking point being the point where the squares make contact. This effect is represented by the first pair of dashed lines in Figure 10b, which diverge in opposite directions. A second mechanism must be assumed to explain why the dividing line does not appear broken. Our hypothesis is that the two lines are rejoined by the following device: The vertical distance of the two sections is adjusted so that the two central ends tally. The outcome of this operation is illustrated by a dashed line made up of horizontal segments and obliques conjoining their extremes. Note that the outcome consists in an overall tilt of the gray line in the direction opposite to Type I vector sum.

Figure 10c illustrates a more complex integration, in which both Type I and Type II effects play a role. The implication of this hypothesis on the "café wall" illusion will be discussed below.

On the basis of the above assumptions, some predictions were made about the summation of the distorting forces and direction of the apparent merging tilt. These predictions have been tested against some patterns, examples of which are shown in the rightmost column of Figure 11. As can be seen, they are more complex versions of the patterns used in Experiment 1, since both the figure and the ground are surfaces with changing luminance. These double variations may be combined in such a way that different pairs of rotatory forces arise, thus allowing observation of their combined effects. As for the stimuli of Experiment 1, a shading vertical stripe was introduced so that the contour deviations were more evident.

The configurations depicted in the first two rows of Figure 11 are illustrative of Type I overall effect. The other two rows illustrate Type II.

Distorting vectors departing from the gray edges are presented in the leftmost column; their direction was determined following the procedure described above: An outline segment shifts toward the region of changing luminance. This operation was repeated whenever the end of an outline segment extended a gray edge. The outcomes of these operations are schematized in the central column of Figure 11, with dashed outlines showing the effects of distorting impulses and local integration.
In this model, the differential strength of shifts toward the white side and the black side has not been taken into consideration. In fact, the present model offers an alternative view to the Figure 7 model of vectors sum. Two opposing forces do not sum their magnitudes; they indeed generate distinct effects that are then integrated. In other words, the same region underlies two distinct misplacements which do not sum as two vectors but integrate as illustrated in Figure 10. This integration proceeds in the same direction when the opposing forces have either the same or different strengths. At this point, it is clear that we have given up accounting for the illusory edge misplacement in terms of vector composition. From now on, the arrows will represent the distorting pulse in the direction stated by the overflow rule.

Some examples of patterns producing these illusions are presented in the two rightmost columns of Figure 11. The two instances chosen to illustrate these types of integration show the effects of two different degrees of overlapping, or ratio length, between outline and fuzzy segments. As one can easily observe, large overlapping (rightmost column) consists in larger fuzzy or outline central segments, whereas small overlapping makes these segments shorter than the lateral ones. These manipulations affect the way the illusory detour appears, since, as we have discussed above, the distorting vectors give rise to abrupt changes of direction when concentrated on short segments and to an edge apparent slant when distributed on larger segments. These differential effects are particularly evident in Figures $11 \mathrm{c}$ and $11 \mathrm{~d}$, where the deformations take the form of a "bottleneck" and a trapezoid ("bell"), respectively.

The four pairs of examples in Figure 11 can thus be classified on the basis of two variables: type of deformation (Figures $11 \mathrm{a}$ and $11 \mathrm{~b}$ correspond to Type I, and Figures $11 \mathrm{c}$ and $11 \mathrm{~d}$ correspond to Type II), and width of deformation region.

The principal aim of Experiment 3 was to test the predictions derived from the hypothesized Type I and Type II local integrations. These predictions are concerned with the direction of apparent convergence of parallel edges and will be checked against the judgments of observers requested to estimate the parallelism versus the convergence of the rectangle edges. The second purpose was to ascertain whether the integration effect had the appearance of a slant or a sharp change of direction. This distinction deserves great attention because it allows specific characteristics of the overall effect to be predicted. We assume that an overall impression of slanting will arise only from local tilt effects. Stepwise effects can also be integrated, but the overall effect will probably appear like a "staircase" rather than a slanted trait. The implications of these assumptions for studies of the café wall illusion are discussed below.

A series of preliminary tests was carried out in order to prepare a task capable of checking the perceived illusory effect. The results indicated that observers have no difficulty in discriminating distortions such as a "bottle- 
a)

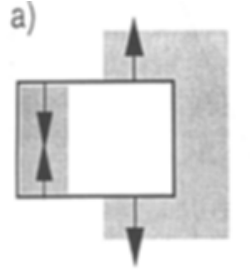

b)

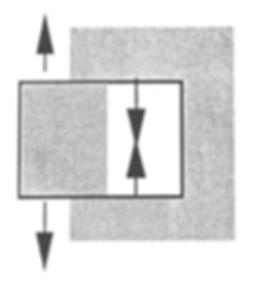

c)
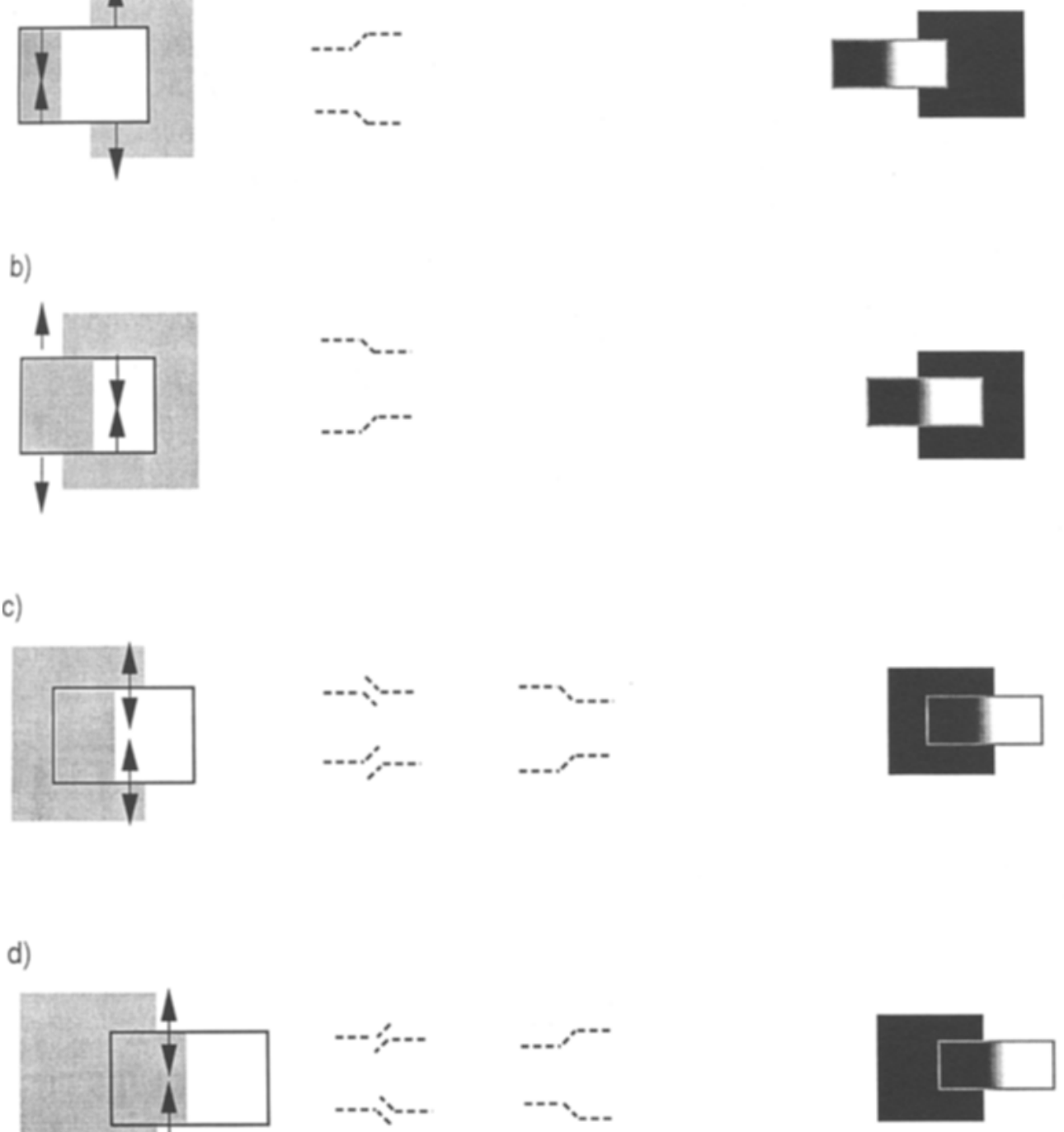

d)
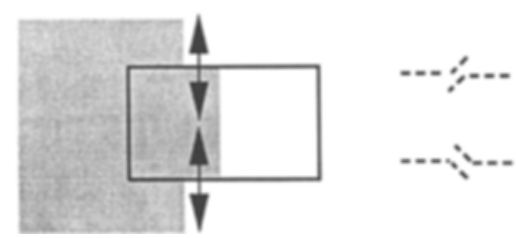
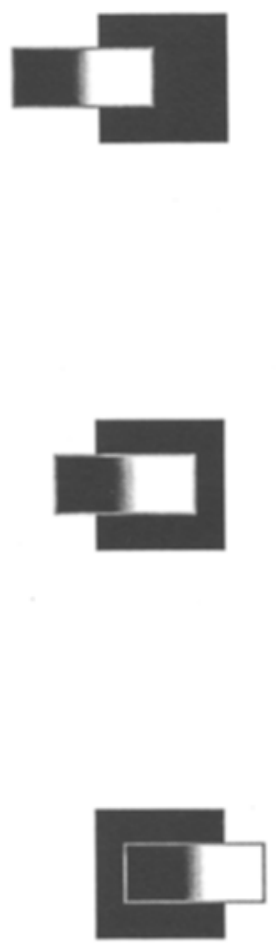

Figure 11. Local distorting effects and the outcomes of local integration processes. In the leftmost column, the distorting forces on the fuzzy edges are schematized. Since the local integration is not affected in direction by difference in strength of the opposing shifts (see text), the same arrow is used to represent the pulses toward the dark side and the light side. The dashed lines in the central columns illustrate the outcomes of the distorting forces and the local integration processes. Two series of patterns illustrate some examples of distortions. For each type of distortion, two examples are presented, which differ in the phase shift of the black-white rectangles.

neck" but are unable to make subtle distinctions between various types of outlines. Consequently, we decided to introduce a recognition task in which observers were asked to check the perceived form against a series of patterns. These included a "bottleneck" and others more similar to trapezoids. These were constructed on the basis of the indications given by the subjects in the preliminary test. A rectangle of the same length as the stimulus test served as a basic pattern for the following deformation: It was vertically divided into halves, the horizontal sides of one half were narrowed so that a thicker rectan- gle resulted, the halves were separated, and the extremes of the horizontal sides were joined by obliques. The distance separating the two halves could have three lengths such that the ratio to total length of the strip was $0.1,0.25$, or 0.75 . It follows that the shortest separation produced a shape similar to a bottleneck, whereas the longest gave a shape very similar to a trapezoid.

\section{Method}

Subjects. Data were collected on 5 subjects ( 2 experienced psychophysical observers and 3 naives). 
Stimuli and Procedure. Some examples of the experimental stimuli can be seen in Figure 12. Each consisted of a horizontal rectangle partially superimposed onto a black area. The rectangle was $0.85 \mathrm{~cm}$ high and $2.2 \mathrm{~cm}$ long framed by a mid-gray line 1 or 2 pixels thick. The inner surface was divided by a vertical shaded bar $(0.21 \mathrm{~cm}$ wide) in a black area and a white area. This blurred space served to magnify the distorting local effects. All the stimulus patterns were derived from two basic ones (corresponding to blackwhite bands in phase or counterphase) by progressive lateral displacement of the smaller rectangle. Nine displacements $(1.1-\mathrm{mm}$ steps) were produced by shifting the rectangle rightward, and nine were produced by shifting in the opposite direction. The underlying surface consisted of a black $3.6 \times 2.6 \mathrm{~cm}$ rectangle. Two sets of configurations were produced simulating black-white bands in phase ( 0 -mm shift) and counterphase (the same line divided black-white on the ground and white-black on the rectangle surface). The 38 stimuli $\left(0^{\circ}\right.$ and $180^{\circ}$ and the 36 showing intermediate phase shifts) were computer-generated (McDraw Plus) and displayed. The experimental pattern appeared on the center of a screen with an image resolution of $800 \times 600$ pixels ( 3 pixels $/ \mathrm{mm}$ ).

Two sets of patterns were constructed: one with a contour thickness of 1 pixel, and another with a contour thickness of 2 pixels. Luminance was $43.8 \mathrm{~cd} / \mathrm{m}^{2}$, approximately the intermediate value be- tween the luminance of black areas $\left(1.93 \mathrm{~cd} / \mathrm{m}^{2}\right)$ and the luminance of white areas $\left(91.1 \mathrm{~cd} / \mathrm{m}^{2}\right)$. The luminance of the white and black regions were measured with a Minolta LS 110. Some shapes were drawn on the top half, from which the subjects could choose the one that best corresponded to their percept: a rectangle and six deformed shapes (three drawn following the procedure illustrated above and the corresponding symmetric; see Figure 12, for some examples).

The subjects were given the following instructions:

Here you can see an example of the figures you should analyse. The figures are made up of two partially overlapping shapes; your task is to examine the horizontal sides of the smaller rectangle and report if they appear as straight or deformed segments. You may observe different types of these deformations: some resembling a sharp change of contour direction, others a gradual bending. We have drawn some outlines from which you can choose the one that bears the strongest resemblance to the contour you perceive. If none of them conform to the configuration displayed, please sketch the deviations you see.

The subjects viewed the stimuli at two different distances (the set with contours 1 pixel thick at $80 \mathrm{~cm}$, and the other set at $170 \mathrm{~cm})$, so that the contour line almost subtended the same visual angle ( $1.41^{\prime}$ and $1.33^{\prime}$ of arc, respectively). Each stimulus was presented twice. The order of stimuli presentation was random and changed for every

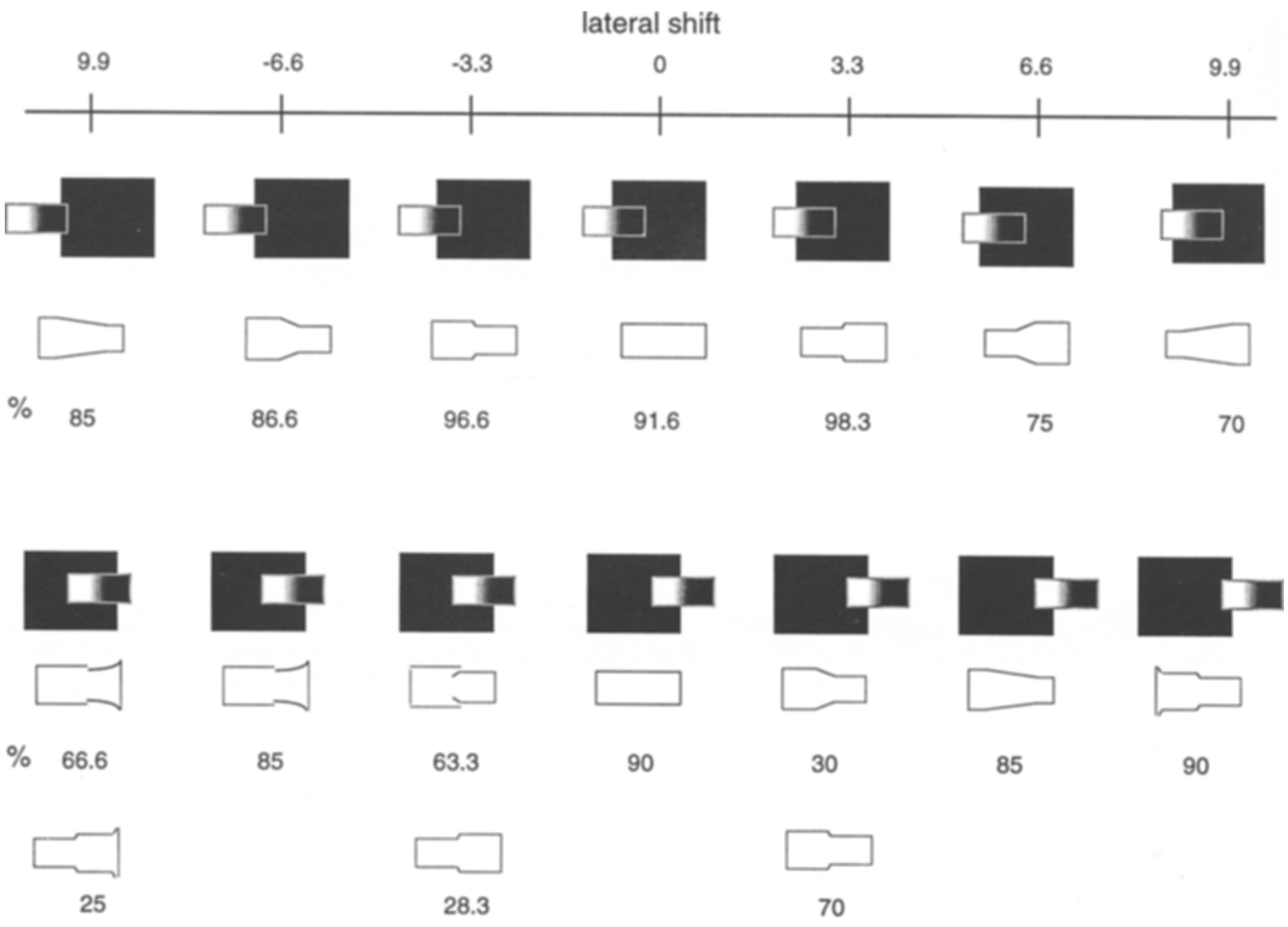

Figure 12. Results of Experiment 3: the most frequently perceived distortions in relation to amplitude of lateral displacement of the rectangular shape. The three leftward and the three rightward displacement groups are represented by the top line. An example pattern is presented in correspondence to the interval marks; below this are presented the perceived shape and the frequency percentage. Line thickness and areas are altered with respect to the stimuli used in Experiment 3. 
subject. During a training session, the subjects were presented with 12 stimuli and were requested to explore different deformations. Sequences of progressive displacements were then shown.

\section{Results}

In Figure 12, the results of Experiment 3 are summed up. The two sets of stimuli (explored at a short distance or a longer distance) yielded very similar results, so the data were pooled. The nine displacements were grouped in three lengths; for each group, the subjects' preferences were computed in order to find the preferred shapes. In the space below the segment indicating the shift width, one of the experimental stimuli, the shape most frequently indicated by the subjects, and the frequency of occurrence expressed as percent of choices are shown.

The results in the upper half of the figure agree with the predictions illustrated in Figure 11 and are drawn from the Type II integration hypothesis. Second, the deformation appears as a sharp change of direction (an outline shift) when the gray edge is short, but it appears as a line slant when the gray edge elongates as a result of wider lateral displacement.

The results reported in the lower half of Figure 11 conform only in part to the predictions. While the directions

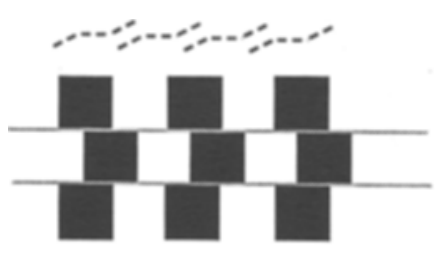

a

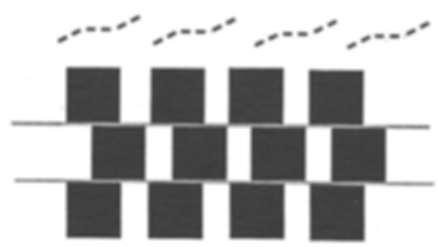

b

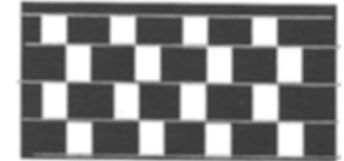

C

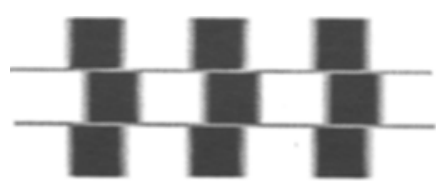

d

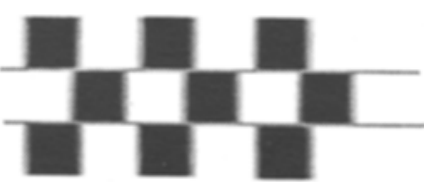

e

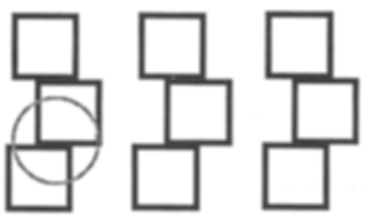

$f$

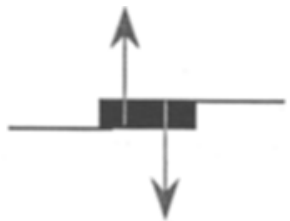

g

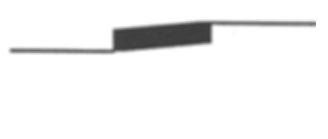

h

Figure 13. (a) The Münsterberg illusion and local distortion of the mortar line. (b) A version of the Münsterberg illusion with white tiles reduced by one half and hypothesized mortar line tilts (dashed lines above). (c) An example of illustration b that magnifies the apparent mortar line slantings. (d) A stepwise distortion of the mortar lines when the phase shift is very low. (e) The effects of phase shift close to $180^{\circ}$. (f) Illustration of the hollow square illusion. (g) Distorting units of the hollow square illusion. (h) The expected outcome of distorting vectors illustrated in g. 
of illusory convergence correspond to those we expected on the basis of the Type I integration hypothesis, some patterns of distortion were not predicted. This occurs when a counterphase shift produces partial overlap of the black areas (second row, leftside figures). Most of the subjects, indicating the black half of the shiftable rectangle, referred to having a clear impression of a "cork," or they observed that the black half appeared as a shape disjointed from the white half, thus seeming to be a hole or an area of the ground. Figure 12 presents the patterns that represent the configurations perceived by such observers. Note, in particular, the widening of the white area and the misalignment of the broken horizontal contours that led to the recognition of the effects of the white irradiation phenomenon. These may be considered demonstrations of what happens when local integration fails. The conditions in which it occurs are unclear, but our findings indicate that the phenomenon is enhanced at longer visual distance and with CRT displays, since, in pilot experiments with paper-drawn stimuli, the white expansion did not appear.

\section{Discussion}

The basic illusory effect and the integration revealed by Experiments 2 and 3 may give an account of well-known optical illusions.

Café wall illusion. The classical illusory pattern is shown in Figure 13: alternate rows of squares shifted by half a cycle and a mortar line of luminance midway between the dark and light tiles. We can easily observe that in this line some outline and fuzzy edges alternate, giving rise to a combination of Type I and Type II integration, as already illustrated in the lowest row of Figure 10. These effects have been represented above the café wall pattern (Figures 13a and 13b) by means of dashed lines illustrating, in the tilted region, the consequences of distorting forces on the fuzzy regions of the mortar line. Each of these fuzzy segments undergoes two opposing impulses located between the BGB and the WGW outlines. It is possible to make the isolated effect of each impulse evident with a simple device. In Figure 13b, the white spaces between black tiles are restricted so that the mortar lines dividing the white tiles disappear and those separating the black regions survive. This implies that the fuzzy segments undergo only one type of distorting effect, that arising from the black flanking outline (BGB). Figure 13c magnifies this illusory tilt originating from Type II vector sum. With the same device, it is possible to make evident the distorting strength that arises from the superimposition of white tiles only.

The local slanted elements represented by the dashed lines in Figure 13 may appear in isolation if a mortar line is absent, as in the second example of the rightmost column of Figure 10. However, when they are parts of the same line, they undergo a process of integration from which two overall effects may emerge: slanting or staircase, as we will illustrate. This theory replicates the one proposed by McCourt (1983), according to which the ap- parent convergence of mortal lines in the café wall illusion arises in two stages. First, the effects of induction produce brightness variations that appear as directional units (i.e., light and dark tilted mortar line segments joining together the corner region of the phase-displaced tile squares of the same luminance). Second, the series of these slanted line elements are integrated by a global process. We disagree with McCourt concerning the local slanted elements identified in a more general phenomenon of line detour than the conjunction of corners in the cafe wall configurations. The mortar line convergence is probably the outcome of a global process of integration that may have different outcomes.

Our hypothesis is that such an overall slant emerges only if local integration produces a slanting line and not an abrupt change of direction. If this is so, the overall effect should vary in relation to some factors. For example, the apparent slant of the mortar line should be affected by the length ratio between the outline and the fuzzy mortar segments. Specifically, very short fuzzy segments should give rise to a stepwise distortion, whereas longer segments should produce an overall impression of slant. Figure 13d illustrates a case in which an outline segment is much longer than the fuzzy one, and the stepwise distortion clearly emerges. When this segment is shortened to a length equal to the fuzzy edge (Figure 13e), the slant effect arises.

Some research has demonstrated that the maximum illusion occurs at a phase shift of $90^{\circ}$ (Moulden \& Renshaw, 1979) or approximately $70^{\circ}$ (Haig, 1989). Why does the strongest effect occur with a phase shift near $90^{\circ}$ ? The findings of Experiment 3 may give an answer. The short lateral shifts in those patterns correspond to limited phase shift in café-wall-like configurations. The results of Experiment 3 demonstrate that, in such conditions, the observed distortions are likely to be the bottleneck type in which abrupt deviations, rather than line tilts, appear. The overall effect is then likely to be a staircase, rather than a mortar line slant. The phase shifts near $180^{\circ}$ in café wall configurations produce local conditions similar to those in the second row of Figure 12, where the results of small counterphase shifts are reported. In these cases, the deformations are also likely to be perceived as sharp changes of direction, though, in some conditions, the sum of opposite deformations gives unclear outcomes. The second important suggestion is given by the results obtained with longer lateral shifts, which give rise to the impression of tilt affecting a region more or less longer than the horizontal sides of the rectangle. The clearer this local impression is, the more likely an overall impression of slanting will arise.

Hollow square illusion (Taylor \& Woodhouse, 1980). The effects seem to be opposite of those observed in the Münsterberg illusion; however, if we observe a detail of Figure $13 \mathrm{f}$, we can easily recognize a familiar pattern inside the circle: two lines each dividing a white area and a neighboring black and white area - that is, a condition in which the line appears to shift toward the black area. 


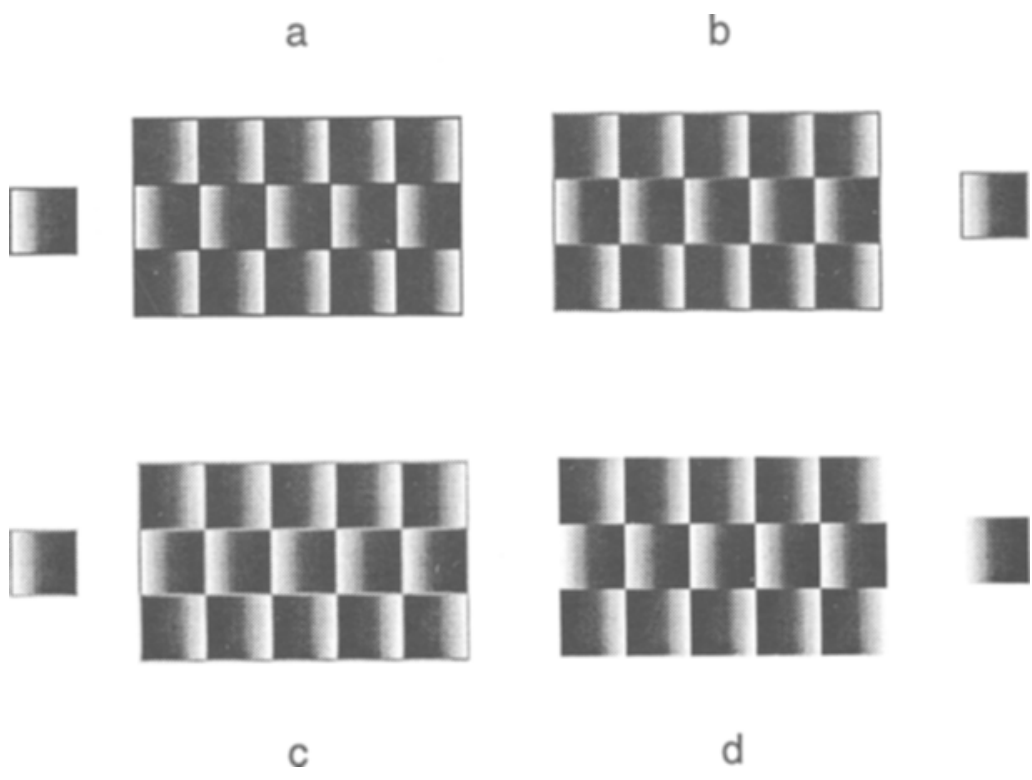

\begin{abstract}
Figure 14. Examples of the lavatory illusion. The tiles in all the configurations have the same gradual changes in brightness. The four configurations differ in mortar line luminance: (a) $90 \%$ black, (b) $50 \%$, (c) $30 \%$, and (d) $0 \%$. Compare the edge distortion of the single tiles beside the configuration and the apparent convergence of the mortar line in the latter.
\end{abstract}

The outcome is illustrated in Figure $13 \mathrm{~h}$ as a counterclockwise tilt of the black outlines from which an overall effect of rightward convergence of the tile rows originates. Bressan (1985) was correct in maintaining that Münsterberg and hollow square illusions were based on the same underlying mechanisms. In light of our results, the mechanism is likely to be the one that Bressan hypothesized in discussing Gregory and Heard's (1979) point of view, even if we can exclude the possibility that it can be reduced to the irradiation of a white area in the direction of a dark one.

Lavatory wall illusion (Woodhouse \& Taylor, 1987). The conditions that produce such an illusion are not clear in the photo reported in Woodhouse and Taylor's article. We have reproduced it in Figure 14, where one can see that distortion occurs only when a mortar line divides the tile rows. Second, if we consider that the tiles in the lavatory wall illusion are identical to the stimuli we used in Experiments 1 and 2, we can conclude that the illusory effect does not require a row of tiles having a smooth brightness variation; rather, it relies on a simpler phenomenon that affects just one single tile.

The lavatory wall illusion is a particular case of distortion similar to those analyzed here. This becomes clear from a comparison of Figures $14 \mathrm{a}, 14 \mathrm{~b}$, and 14c, containing mortar lines of different luminance: black first, mid-gray second, and light gray third. The horizontal mortar lines bordering the central row appear to converge rightward in Figure 14a, leftward in Figures $14 \mathrm{~b}$ and 14c. They appear as parallel in Figure 14d, where the mortar line is absent. This apparent mortar line tilt is congruent with the predictions drawn from the rule stating that a sharp trait is deviated toward the region of highest or lowest luminance. Note that the apparent convergence of the edge tiles may be different when perceived in isolation or in a wall.

\section{CONCLUSION}

The phenomenon described here has different outcomes. It consists of a reliable error in the perceived position of gray edges, which may appear shifted or tilted with respect to a gray outline. Phenomena of gray (or blurred) edge misplacement are well known, but the illusion we illustrated appears to be caused by processes or factors different from those assumed by the current models of edge localization. We have demonstrated that the direction of the distortion is not predictable on the basis of luminance arrangement or contrast of the gray edge, because such distortion changes its polarity according to the luminance profile of an outline reference. For example, a vernier alignment task would not be proposed, because the same gray edge would be displaced toward its dark phase (thus in agreement with the irradiation hypothesis) when the observer is requested to align it with a gray outline against a white background and toward the light phase when the gray outline is printed against a black background.

The account we have offered is obviously incomplete since we have not found the underlying phenomenon that generates the misperception described by the overflow rule. The only certain datum is that the illusory shift moves toward the region changing in luminance. An understand- 


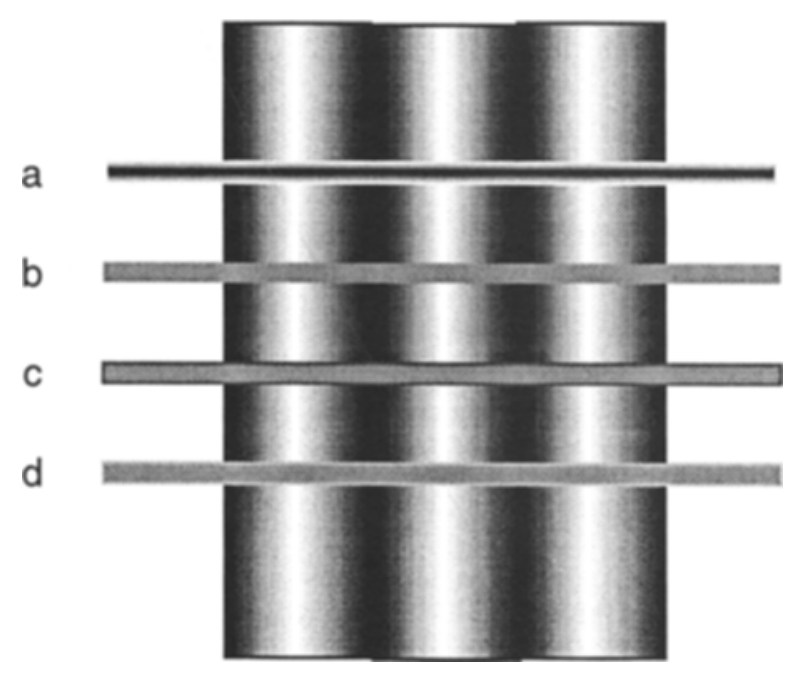

Figure 15. Apparent distortions produced by an induction grid on strips of different area and contour luminance.

ing of the way in which luminance differences affect alignment relations has led us to reexamine McCourt's (1982, 1983) works on the café wall illusion and the brightness induction effect, because, among the induction grating phenomena, some interesting illusions emerge.

In Figure 15, a triangular wavegrating and various induced strips are presented. The first illusory phenomenon to note can be seen in strip a. It consists of a half-period triangular wave (symmetrical blurred strip) perpendicular to the orientation of the inducing grating. Note the difference between the external region of the strip and the one traversing the grating. The latter appears to widen and shrink at regular periods corresponding to the induction grating phases. More important is the correspondence between the peaks of the induction grating and the illusory wave inside the induced field. The point of maximum gray expansion is perceived in correspondence to the white peak of the inducing wave - that is, in a region where the dark-induced field shades into the white ground. If we move back or forward half a period, the profile of luminance changes because the region in which the gray strip fades into white is bordered by the black edge of the wavegrating. These two profiles of luminance are very similar to the profiles correspondent to the gray edge and the outline contour, respectively. This offers convincing proof that a homogeneous field appears to expand when its contour changes from outline into blurred or gray edge.

It can be demonstrated that the effects of grid induction emerge not only as periodic widening of the inner area but also as an apparent waving of the contour. Strip b is the mid-gray-induced field studied by McCourt (1982), in which the counterphase-induced brightness induction can be observed. In strip c, the same strip is bordered by a dark gray line; in strip d, the contour is light gray. The two last strips show brightness variations similar to strip b, but, in addition, the horizontal edges appear bordered by waving lines. Note that the illusory waving in strip $d$ is counterphase with respect to strip c. If we inspect the borders separating the inducing and induced fields, we can observe that the peak of apparent expansion is perceived in the correspondence of the inducing peaks: white when the contour line is light gray, and black when the contour line is dark gray. That is, the gray-induced strip bores into the ground when bordered by a blurred edge. Strips $c$ and $d$ make it evident that the brightness induction phenomena may result in a contour distortion of the induced field and that the conditions for this to occur are very similar to those we produced in the present research to magnify the illusion illustrated in Figure 1. Our findings do not explain these distortions. However, the final observations make an important suggestion concerning the path to take, since the illusion is likely to belong to the process of brightness induction extensively studied by McCourt (1982).

Our experiments raise a final question. The magnitude of the edge misperception analyzed here is likely to depend on the thickness of the gray line. No measurement of this dependency has been carried out here, but we are led to expect enhancement of the illusion, and perhaps changes in direction, when seen at small visual angles. Some research supports this hypothesis. Gregory and Heard (1979) demonstrated that the café wall illusion decreases with an increase in mortar line width (from $1^{\prime}$ to $12.9^{\prime}$ of arc subtended by the eye). Zaidi (1989), in a study of visual grating induction, demonstrated that cohesive induced grating appears when small visual angles separate the inducing edges, but, with larger angles, the induced field appears as "light and dark patches." These variations in the cohesiveness of the induced field may generate differences in the apparent misplacement of the gray edge in the present illusion. Finally, it may be considered that, at longer viewing distances, some abrupt changes in direction of mortar line, such as those appearing in Figure 13d, are likely to disappear, thus giving rise to an overall integration of single steps such as that hypothesized by McCourt.

\section{REFERENCES}

Bex, P. J., \& EDGaR, G. K. (1996). Shifts in the perceived location of a blurred edge increase with contrast. Perception \& Psychophysics, 58, $31-33$.

Bressan, P. (1985). Revisitation of the family tie between Münsterberg and Taylor-Woodhouse illusions. Perception, 14, 579-585.

Gregory, L. R., \& Heard, P. (1979). Border locking and the café wall illusion. Perception, 8, 365-380.

Gregory, L. R., \& HEARD, P. (1983). Visual dissociation of movement, position and stereo depth: Some phenomenal phenomena. Quarterly Journal of Experimental Psychology, 35A, 217-237.

HaIG, N. D. (1989). A new visual illusion, and its mechanism. Perception, 18, 333-345.

KITAOKA, A. (1998). Apparent contraction of edge angles. Perception, 27, 1209-1219.

Mather, G., \& Morgan, M. (1986). Irradiation: Implications for theories of edge localization. Vision Research, 26, 1007-1015. 
MCCourt, E. M. (1982). A spatial frequency dependent grating-induction effect. Vision Research, 22, 119-134.

McCourt, E. M. (1983). Brightness induction and the café wall illusion. Perception, 12, 131-142.

Morgan, M. J., \& AiBa, T. S. (1985). Vernier acuity predicted from changes in the light distribution of the retinal image. Spatial Vision, 2, 150-161.

Morgan, M. J., \& Moulden, B. (1986). The Münsterberg figure and twisted cords. Vision Research, 26, 1793-1800.

Moulden, B., \& Renshaw, J. (1979). The Münsterberg illusion and irradiation. Perception, 8, 275-301.

NaIman, A. C., \& Makous, W. (1993a). Information transmission for sub-pixel edge positioning. Journal of the Society for Information Display, 1/4, 437-447.

Naiman, A. C., \& Makous, W. (1993b). Undetected gray strips displace perceived edges nonlinearly. Journal of the Optical Society of America A, 10, 794-803.

Pierce, A. H. (1898). The illusion of the kindergarten patterns. Psychological Review, 5, 233-253.

TAYLOR, S., \& WOODHOUSE, J. M. (1980). A new illusion and possible links with the Münsterberg and Fraser illusions of direction. Perception, 9, 479-481.

Tiraboschi, M., \& Roncato, S. (1997). Illusione di Münsterberg e unità direzionali. In A. Cavedon \& L. Zanuttini (Eds.), Tra percezione e arte. Scritti in ricordo di Aurora Bazzeo. Padua: Il Poligrafo.

WATT, R. J., \& MoRGan, M. J. (1983). The recognition and representation of edge blur: Evidence for spatial primitives in human vision. $V i$ sion Research, 23, 1465-1477.

Woodhouse, J. M., \& TAYloR, S. (1987). Further studies of the café wall and hollow squares illusions. Perception, 16, 467-471.
ZAIDI, Q. (1989). Local and distal factors in visual grating induction. Vision Research, 29, 691-697.

\section{NOTES}

1. Some researchers on the Münsterberg illusion have studied the distorting effects of diagonally aligned squares on a line traversing their adjacent corners. This particular configuration is obtained by combining white tiles and shorter black tiles in alternate rows. A particular phase shift allows one to perceive an illusory slant of the mortar line when the black tiles have no overlapping but only a corner contact $(\mathrm{Ki}-$ taoka, 1998; Pierce, 1898; Tiraboschi \& Roncato, 1997). This configuration may be considered a particular version of the Münsterberg illusion in which only the white tiles overlap. In fact, the difference in length of tiles does not prevent the formation of a pattern (two black tiles and a right line conjoining the upper side of one with the lower side of the other) that, according to some researchers, may be considered one of the sources of the Münsterberg illusion. The hypothesis that the diagonally aligned squares may generate illusory tilt effects deserves consideration, yet it cannot account for the apparent slanting of the gray line when bordering one square only, as Figure $1 \mathrm{~d}$ demonstrates.

2. The experiments presented here were the exact replicas of experiments carried out with printed stimuli. The magnitude of the distorting effects registered here was higher than the illusion magnitudes registered with the printed stimuli, although all qualitative effects were replicated.

(Manuscript received January 22, 1998; revision accepted for publication May 11, 1999.) 